\title{
Hypothèses sur l'origine et la diffusion du complexe rituel du tlacaxipehualiztli
}

Anne-Marie Vié-Wohrer

\section{OpenEdition}

1 Journals

Édition électronique

URL : https://journals.openedition.org/jsa/10602

DOI : 10.4000/jsa. 10602

ISSN : 1957-7842

Éditeur

Société des américanistes

Édition imprimée

Date de publication : 20 décembre 2008

Pagination : 143-178

ISSN : 0037-9174

\section{Référence électronique}

Anne-Marie Vié-Wohrer, «Hypothèses sur l'origine et la diffusion du complexe rituel du

tlacaxipehualiztli », Journal de la Société des américanistes [En ligne], 94-2 | 2008, mis en ligne le 16 novembre 2013, consulté le 03 septembre 2022. URL : http://journals.openedition.org/jsa/10602 DOI : https://doi.org/10.4000/jsa. 10602 


\title{
HYPOTHÈSES SUR L'ORIGINE ET LA DIFFUSION \\ DU COMPLEXE RITUEL DU TLACAXIPEHUALIZTLI
}

\author{
Anne-Marie VIÉ-WOHRER *
}

\begin{abstract}
Nous proposons, dans cet article, de dépasser les limites géographiques et culturelles du Mexique central ancien pour réfléchir sur un complexe rituel et sacrificiel donné. Ce complexe, mis en scène une fois par an dans tout l'empire mexica, était célébré en l'honneur du dieu Xipe Totec. Il était qualifié de "Fiesta general », selon les sources les plus importantes du $\mathrm{XvI}^{\mathrm{e}}$ siècle. Certains des rituels qui y étaient mis en œuvre ressemblent, au moins dans leur exécution, à d'autres pratiqués dans certaines aires culturelles du territoire nord-américain. L'examen de ces derniers contribue à mieux comprendre le complexe mésoaméricain lui-même. [Mots clés : sacrifice, rituel, écorchement, Mexique.]
\end{abstract}

Hipótesis sobre el origen y la difusión del complejo ritual del tlacaxipehualiztli. La autora trata de explorar similitudes que existen entre unos rituales practicados por grupos establecidos al norte de Mesoamérica, sobre todos recolectores-cazadores, y otros famosos en el ámbito mexica. [Palabras claves: sacrificio, ritual, desollamiento, México.]

Hypothesis about origins and diffusion of the ritual process of tlacaxipehualiztli. This article tries to document similarities between rituals practised mostly by huntergatherers of the North of Middle America and one of the most famous Aztec ritual complex. [Key words : sacrifice, rituals, flaying, Mexico.]

À l'époque postclassique, parmi les populations du Mexique central et, en partie, méridional, deux rituels sacrificiels étaient pratiqués. La mise à mort de la victime, qui avait lieu lors des dernières étapes de l'accomplissement de ces rituels, consistait, pour le premier, appelé tlacaxipehualiztli (« écorchement des hommes »), à arracher le cœur, puis à écorcher le corps ; pour le deuxième, appelé tlacacaliliztli (" envoyer des dards sur quelqu'un »), à percer le corps de la victime de flèches ou de javelines.

* École du Louvre/université de Paris VIII, Saint-Denis, Palais du Louvre, Porte Jaujard, place du Carrousel, 75038 Paris cedex 01 [Awohrer@aol.com].

Journal de la Société des Américanistes, 2008, 94-2, pp. 143-178. (O Société des Américanistes. 
Ces deux formes rituelles de mise à mort, comme dans toute stratégie sacrificielle comprise dans son acception élargie de meurtre rituel ${ }^{1}$, apportaient des bénéfices d'ordre personnel (ascension sociale, acquisition de richesses...) pour ceux qui avaient fourni les victimes et d'ordre général pour la communauté. Les rituels étant exécutés en faveur de divinités responsables du renouveau, les bénéfices que la communauté en attendait étaient vitaux, puisque de l'exécution des rituels dépendait la survie du groupe.

Ces rituels étaient accomplis lors de trois fêtes annuelles du calendrier solaire. La première, qui avait lieu pendant le deuxième mois ${ }^{2}$ appelé tlacaxipehualiztli (comme le rituel lui-même), marquait la fin de la saison sèche et le début du printemps, quand les premières plantes commencent à sortir de terre. La deuxième fête, qui correspondait au douzième mois appelé ochpaniztli ${ }^{3}$, marquait la pleine saison des pluies, quand le maïs atteignait la belle maturité et pouvait commencer à être récolté. La troisième fête, appelée izcalli ${ }^{4}$, avait lieu pendant le dernier mois de l'année du même nom, le dix-huitième, en pleine saison sèche.

Les deux premiers rituels étaient exécutés non seulement à Tenochtitlan, capitale de l'empire, et dans ses provinces, mais aussi dans les royaumes indépendants situés soit à l'intérieur, soit à l'extérieur de l'empire ${ }^{5}$. Selon Diego Durán (1967, I, pp. 95-135), la fête de tlacaxipehualiztli était considérée comme une "Fête générale » dans tout l'empire, spectacle sacré auquel tous étaient contraints d'assister.

Parmi les divinités célébrées au cours de ces deux fêtes, il y en avait à caractère agraire proprement dit comme la déesse du maïs mûr Chicomecoatl. Lors de la fête d'ochpaniztli, une femme personnifiant cette déesse était immolée et écorchée, au Cinteopan (« Le temple de l'épi de maïs »; Torquemada 1969, II, pp. 8, 152, 155). Un prêtre du temple revêtait alors sa dépouille, arborant des épis de maïs dans les mains et dans la coiffure, et allait siéger en majesté, au milieu des différents représentants des dieux de la pluie et du maïs (Codex Borbonicus, 1991, pp. 29-30).

D'autres divinités investies de fonctions agraires (représentées dans des manuscrits ou dans des sculptures) revêtaient aussi en partie ou en totalité la peau d'une victime sacrificielle. Le visage de Cinteotl, dieu du maïs jeune, était en partie caché sous un demi masque découpé dans la peau de la cuisse d'un sacrifié ; le corps de Tlazolteotl, déesse de la terre, de la fécondité et du péché de la chair, était entièrement revêtu d'une dépouille humaine.

Les dieux Xipe Totec et Camaxtli (son homologue tlaxcaltèque ${ }^{6}$ ) revêtaient aussi une dépouille complète. Les deux étaient investis de fonctions agraires qui semblent avoir été complétées par une fonction guerrière chez Xipe Totec à Tenochtitlan ${ }^{7}$ et par une fonction cynégétique chez Camaxtli à Tlaxcala. Dans les manuscrits, ce dieu de la chasse est représenté revêtu soit d'une peau écorchée, soit d'une peinture corporelle à raies blanches sur fond rouge, attribut des 
condamnés au sacrifice du tlacaxipehualiztli et en particulier lors de l'un de ses épisodes, le tlahuahuanaliztli, appelé « sacrifice du gladiateur » par les Espagnols (Durán 1967, I, fig. 13) ${ }^{8}$.

L'on écorchait aussi des victimes en l'honneur du dieu du feu, Xiuhtecuhtli, tous les quatre ans à Cuauhtitlan, pendant la fête d'izcalli ${ }^{9}$. Deux esclaves de sexe féminin étaient décapitées puis écorchées; deux seigneurs qui revêtaient leurs dépouilles dansaient, tenant à la main l'un de leurs fémurs.

\section{LES RITUELS SACRIFICIELS}

\section{Le tlacaxipehualiztli}

Ce rituel a été abondamment commenté dans les sources du $\mathrm{XVI}^{\mathrm{e}}$ siècle. Il avait lieu après l'immolation classique, qui consistait à arracher le cœur de la victime sur la pierre de sacrifice, tout de suite après pour les victimes ordinaires et à l'issue du tlahuahuanaliztli pour les victimes plus valeureuses. Des officiants écorchaient la peau du corps, des bras et des cuisses, en la soulevant de part et d'autre d'une longue incision réalisée au milieu du dos. Les mains restaient à demi attachées aux bras, le visage et une partie du cuir chevelu étaient écorchés comme un scalp. La peau du corps était utilisée comme un vêtement avec des manches et des jambes de pantalon que l'on enfilait. La peau du visage et du cuir chevelu servait de masque facial et constituait une coiffure élaborée, attachée en chignon à l'arrière de la tête (Figure 1).

La victime était ensuite décapitée, son corps dépecé, la cuisse (morceau de choix) étant réservée au grand tlatoani. Vers la fin du sacrifice avait lieu une sorte de ronde, le motzontecomaitotia (" danse avec des têtes »), avant que les têtes ne soient accrochées au tzompantli, présentoir à crânes. Ce qui restait du corps du sacrifié était récupéré par la famille de celui qui avait capturé la victime. Les morceaux étaient consommés sous forme de tlacatlaolli (« ragoût d'homme »), plat composé de chair cuite et de maïs. Tous les membres de la famille en mangeaient, à l'exception de celui qui avait capturé le prisonnier; car la capture établissait entre eux des liens d'une filiation symbolique. Un des fémurs du sacrifié, après avoir été orné de bannières en papier, était attaché, bien en vue, comme un trophée, à un mât prévu à cet effet et érigé à la porte de la demeure de celui qui avait capturé le guerrier. Cette relique était appelée malteotl, « dieu captif ».

\section{Le tlacacaliliztli}

Au contraire du tlacaxipehualiztli, ce rituel est peu commenté dans les sources du $x I^{e}$ siècle. En revanche, il apparaît dans de nombreux manuscrits pictographiques provenant de différentes régions : cuicatèque comme les codex Fernández Leal (pl. 6, 10) et Porfirio Díaz (pl. $\mathrm{K}^{*} \mathrm{v}^{\circ}, \mathrm{Pv}^{\circ}$ ) (in Códices cuicatecos 2001), 

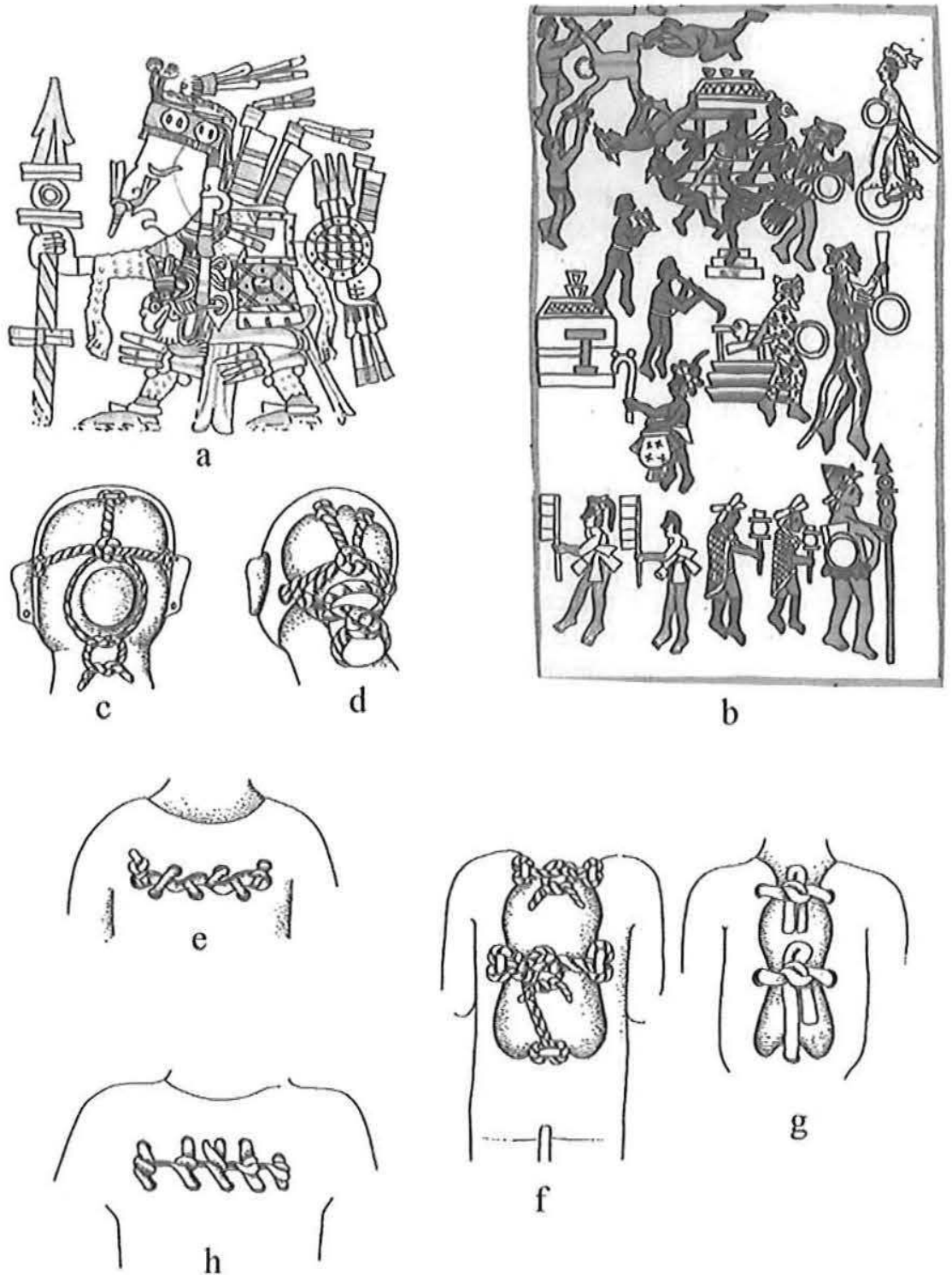

FIG. 1 - Sacrifice du tlacaxipehualiztli:

(a) Le dieu Xipe Totec (d'après Codex Borgia, p. 25) ;

(b) fète du Tlacaxipehualiztli (d'après Primeros Memoriales 1905) ;

(c, d, e, f, g, h) exemples d'utilisation de la peau écorchée (dessins de Françoise Bagot). 
mixtèque comme les codex Nuttall et Becker I, ainsi que d'autres manuscrits du Mexique central ${ }^{10}$. Ce rituel figure souvent aux côtés d'une représentation du tlahuahuanaliztli. Dans ces représentations, le sacrifié, attaché à une sorte de chevalet, est percé de flèches ou de dards ; son sang s'écoule sur une représentation de la terre qu'il féconde (Figure 2).

Des rituels comparables, l'un au tlacaxipehualiztli, l'autre au tlacacaliliztli, étaient exécutés à Cuauhtitlan tous les quatre ans (Motolinía 1996, pp. 189-191), lors de la fête d'izcalli célébrée en l'honneur du dieu du feu, Xiuhtecuhtli. Au cours du premier rituel qui avait lieu la veille du sacrifice considéré comme principal, deux femmes esclaves étaient égorgées, décapitées, écorchées, en partie dépecées et leurs fémurs retirés des cuisses. Le lendemain, des notables revêtaient les dépouilles et couvraient leur visage d'un masque découpé dans la peau. Au cours du second rituel, une multitude d'archers décochaient des flèches sur un groupe de six captifs de guerre, dont chacun était hissé en haut d'un mât. Dès que les captifs blessés tombaient des mâts, on leur arrachait le cœur, on les égorgeait, puis on les décapitait. Ils étaient remplacés par d'autres groupes de six captifs auxquels le même sort était réservé. Les corps étaient confiés à des officiants qui, à leur tour, les remettaient à des notables pour qu'ils soient consommés.

En résumé, au moment de l'arrivée des Espagnols, il existait en Mésoamérique des rituels sacrificiels très élaborés et très complexes ; ils étaient particulièrement exécutés pour les divinités de la végétation et de la fécondité comme Chicomecoatl, Tlazolteotl, Cinteotl, Xipe Totec (ce dernier étant lié à la guerre), ainsi que pour celles de la chasse comme Camaxtli et du feu comme Xiuhtecuhtli. Ces rituels avaient lieu au cours de fêtes fixes du calendrier solaire. Leurs traits les plus caractéristiques étaient :

- Le caractère public et spectaculaire du sacrifice auquel tout le monde était obligé d'assister.

- La variation du statut des sacrifiés selon les divinités auxquelles on les sacrifiait. Dans le cas de Xipe Totec, il y avait deux genres de sacrifiés. Pour le sacrifice « ordinaire », celui qui n'était pas précédé du « combat du gladiateur », la victime était un esclave ; mais pour le sacrifice qui était précédé de ce combat, la victime était toujours un prisonnier de guerre valeureux. Dans les deux cas, la victime avait droit à de nombreux égards pendant la période qui précédait sa mise à mort. Pour les sacrifices aux autres divinités de la végétation et, en particulier, pendant la fète d'ochpaniztli, les victimes étaient des jeunes filles dont l'origine n'est pas spécifiée. C'était un homme jeune particulièrement robuste qui revêtait leur dépouille.

- La relation spécifique entre celui qui capture et la victime. L'homme qui avait pris le captif, dans le cas du tlacaxipehualiztli (précédé du tlahuahuanaliztli) et du tlacacaliliztli, était toujours un guerrier. La capture créait des liens de parenté, d'affection et de propriété. Le « parrain » assistait à l'immolation de 


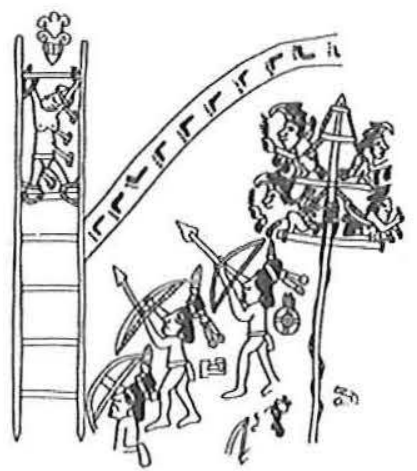

a
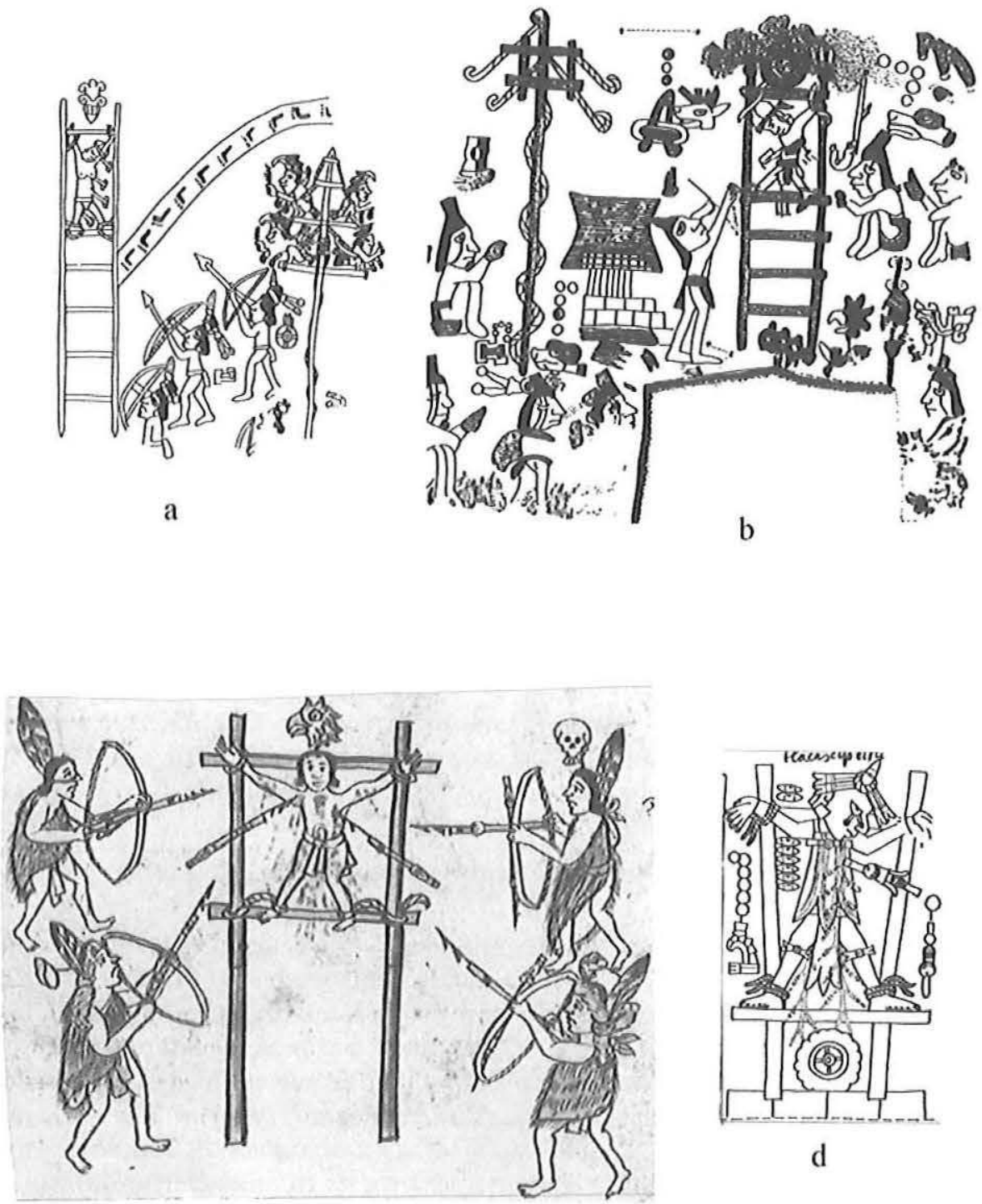

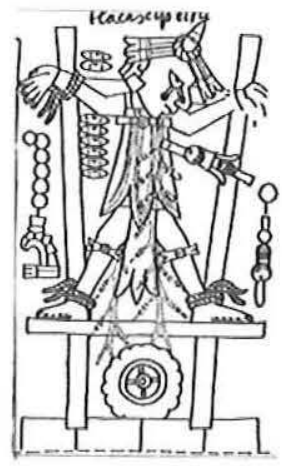

d

$\mathrm{c}$

FIG. 2 - Sacrifice du tlacacaliliztli :

(a) d'après Codex Fernández Leal (pl. 10);

(b) d'après Codex Porfirio Diaz (pl. $\mathrm{P}^{*}$ );

(c) d'après Historia tolteca chichimeca (f. 28r, in Vié-Wohrer 1999, II, pl. A 42 f);

(d) d'après Codex Nuttall (p. 84). 
son prisonnier sans y participer, mais en exprimant des sentiments de tristesse. Il était le propriétaire de la peau du prisonnier qu'il prêtait, tous les jours, pendant un mois. Au bout d'un mois, la peau qui avait cessé de lui appartenir était enterrée dans une cache au bas du temple de la divinité.

- La mise à mort. La mort était donnée soit par arrachement du cœur réalisé par des officiants du temple, soit par décochage de flèches ou de javelines sur la victime immobilisée. Dans ce dernier cas, le sacrifié était attaché à une sorte de chevalet ou à un mât. Tout un groupe d'archers participait à son exécution. Comme à la chasse, le cour de la victime était visé en premier lieu.

- Le sort du corps après la mort. Les sources ne nous informent à ce sujet que pour le tlacaxipehualiztli et la fête d'izcalli à Cuauhtitlan. Le corps, ainsi que le visage et une partie du cuir chevelu, est écorché, puis dépecé. Un fémur est conservé par celui qui a capturé le sacrifié, la tête est coupée et accrochée au tzompantli dans le centre cérémoniel. Le sang est recueilli dans un récipient et offert aux dieux : nombreuses sont les statues qui en sont ointes. La peau est prêtée à des dévots ou à des pauvres qui la revêtent pendant le mois, pour demander l'aumône ou à des fins curatives, Xipe étant censé guérir des maladies de la peau. Le port de la peau, ainsi que celui des attributs principaux de la divinité, conférait à son porteur un statut temporaire de divinité (Durán 1967, I, p. 97). À la fin du mois, les peaux étaient enterrées et ainsi «conservées » au bas du temple de Xipe Totec. La chair, après avoir été dépecée, revient à la famille de celui qui a capturé le sacrifié, à l'exception de la cuisse, pièce réservée à l'empereur. Elle est cuisinée, comme n'importe quel mets, et consommée. Nous l'avons dit, la parenté fictive qui s'établit entre le captif et celui qui l'a capturé empêche ce dernier de toucher au repas.

L'étude de ce rituel sacrificiel soulève depuis longtemps de nombreuses questions relatives à sa diffusion, sa signification et son origine. Miguel Acosta Saignes (1950) avait cherché des réponses à ces questions, mais il s'était surtout intéressé à la diffusion du rituel. Il avait dressé une carte de sa distribution géographique dans différentes régions du Nouveau Monde, en Amérique centrale notamment, chez les Nicarao, descendants de groupes de langue nahua émigrés depuis le Mexique (Fowler 1989, pp. 12, 32-49) dans la région nommée par Gonzalo Fernández de Oviedo ${ }^{11}$ au $\mathrm{xvl}^{\mathrm{e}}$ siècle, Provincia de los Desollados. Acosta Saignes avait aussi repéré des traces de ce rituel chez les Caribes des Antilles et du Venezuela, ainsi qu'en Amérique du Sud, en Colombie, en Équateur et au Pérou. Il en avait aussi signalé la présence en Amérique du Nord, mais d'une façon moins précise. Il estimait, par ailleurs, que l'origine, tant de l'écorchement (et du dépècement) que de l'arrachement du cœur, devait remonter à des populations de chasseurs-cueilleurs habiles dans le dépècement des animaux.

Pour compléter et élargir cette première étude, il convenait de s'intéresser au nord de la Mésoamérique et même aux régions situées au nord du Mexique 
actuel. Une révision des données de ce vaste territoire a donc été entreprise, non seulement parmi les populations soumises par les Espagnols au $\mathrm{XvI}^{\mathrm{e}}$ siècle, mais aussi parmi celles qui ne furent colonisées que bien plus tard et ont conservé la plupart de leurs traits culturels jusqu'à leur soumission définitive ${ }^{12}$. Pour ces derniers, les récits des Européens, en particulier français, qui les ont découverts aux $\mathrm{XVII}^{\mathfrak{e}}$ et $\mathrm{XVIII}^{\mathrm{e}}$ siècles, sont d'un grand profit. Ce sont des témoignages de première main sur leur culture en général et sur leurs croyances et leurs rituels en particulier ${ }^{13}$.

Parmi ces populations, nous ne retiendrons que les plus importantes en les abordant d'ouest en est. La plus occidentale est celle des Anasazi, suivis dans l'État du Texas par d'autres groupes: Caddo, Tonkawa, Apaches Lipan et Karankawa, qui se rattachent à des aires culturelles différentes. À l'extrémité orientale de cet État, dans la vallée inférieure du Mississippi, étaient établis les Natchez que l'on situe traditionellement dans l'aire culturelle des «Eastern Woodlands ». Plus au nord, au Nebraska - aire culturelle des " Plaines » - se trouvaient les Pawnee (Griffin-Pierce 1995, p. 66). Nous avons retenu ce groupe malgré son grand éloignement pour deux raisons : il était apparenté aux Caddo et aurait quitté l'Est du Texas dont il aurait été originaire vers 1300 de notre ère.

Le choix des groupes que l'on vient de citer repose sur deux grands arguments.

Le premier est l'existence, dans cette vaste région, d'un continuum linguistique. Certaines des langues parlées dans le Sud-Ouest et dans une partie des "Plaines" (aire qui s'étend jusqu'au Texas) se rattachent à la famille utoaztèque ${ }^{14}$. En second lieu, et c'est là un point tout aussi important que le premier, les groupes retenus mettaient tous en scène l'exécution de leurs prisonniers et pratiquaient éventuellement l'anthropophagie rituelle. Peut-être partageaient-ils donc certains traits avec le complexe tlacaxipehualiztli-tlacacaliliztli mésoaméricain?

Pourtant, en raison des différences de complexité entre les cultures de ces groupes et celles des populations mésoaméricaines, sacrifices, mises à mort et anthropophagie ont peu retenu l'attention chez eux et ont souvent été pris pour de simples expressions de violence, vides de contenu religieux. On peut toutefois analyser ces faits en les rattachant au concept élargi de sacrifice tel que défini par Valeri ${ }^{15}$.

L'examen auquel nous procéderons ci-dessous est organisé en trois parties concernant soit une portion d'aire culturelle, soit une entité territoriale (comme le Texas) où les groupes, bien que géographiquement proches, se rattachent à des aires culturelles distinctes. Dans chaque cas, nous nous intéresserons au contexte dans lequel apparaissent les éléments du complexe rituel, puis aux éléments eux-mêmes et, finalement, aux correspondances que l'on peut établir avec le rituel sacrificiel mésoaméricain. Les données dont nous disposons sont d'origines diverses et de valeurs inégales. Elles sont d'ordre archéologique pour le SudOuest et les Eastern Woodlands et d'ordre historique et ethnologique pour les 
autres groupes qui ont conservé l'essentiel de leur culture jusqu'à une époque relativement récente.

\section{Les ANasazi du Sud-Ouest}

Parmi les nombreux groupes qui habitaient cette région à cheval entre les États du Colorado, de l'Utah, du Nouveau Mexique et de l'Arizona, le plus important était celui des Anasazi (Figure 3). Ce groupe, désigné au xvi siècle sous le nom de Pueblo par les Espagnols parce qu'ils vivaient dans des villages, existait comme tel depuis au moins deux mille ans (Brody 1993, p. 41) ${ }^{16}$. En 1530, au moment du contact avec les Espagnols, six langues étaient parlées par les Pueblo ${ }^{17}$. Elles sont classées en deux groupes principaux en partie liés ${ }^{18}$, le groupe kiowa-tanoan parlé à l'est et le groupe uto-aztèque ${ }^{19}$ parlé à l'ouest. Au sud-ouest de cette région, il existe une langue indépendante, le zuni.

Des populations de chasseurs-cueilleurs vécurent d'abord dans ce secteur; descendant de la tradition du "désert archaïque ». Dès le $\mathrm{II}^{\mathrm{e}}$ millénaire avant notre ère, elles cultivaient cependant certaines plantes sauvages et construisaient de sommaires systèmes d'irrigation (ibid., pp. 40-41). Vers le $\mathrm{I}^{\mathrm{er}}$ millénaire avant notre ère, apparaît la culture d'un maïs primitif et de cucurbitacées (ibid., p. 68) ${ }^{20}$. C'est seulement vers le viI ${ }^{\mathrm{e}}$ siècle de notre ère que l'agriculture devient la principale source d'aliments ${ }^{21}$, alors que la chasse et la cueillette continuent à être pratiquées. De nos jours encore, la chasse (même si le gibier est moindre) reste importante : des rituels cynégétiques sont encore exécutés, sous forme de pèlerinages auprès d'autels constitués de bois de cerfs, dans des sanctuaires fréquentés depuis six siècles (ibid., p. 208, fig. 122).

Ces populations entretenaient des relations de troc non seulement avec leurs voisins immédiats, mais aussi avec des groupes plus éloignés. Parmi ces derniers, il y avait les agriculteurs des Woodlands du Sud-Est, les chasseurs des Plaines, les agriculteurs du Nord de la Mésoamérique, ainsi que les nomades des alentours. Les Anasazi échangeaient des produits agricoles, des tissus, des vanneries, des turquoises et des coquillages contre des peaux de bisons et d'autres produits ${ }^{22}$.

Les Anasazi, devenus bâtisseurs de villages, de villes et de palais à partir de 900 de notre ère, ont laissé des centaines de sites archéologiques (dont certains ont été occupés pendant plusieurs siècles), en particulier dans la région de Chaco Canyon (au nord-ouest du Nouveau Mexique) qui semble avoir été le centre de leur rayonnement culturel. L'âge d'or de leur civilisation se situe entre le $\mathrm{x}^{\mathrm{e}}$ et le $\mathrm{XIII}^{\mathrm{e}}$ siècles. Une première période (950-1150) est marquée par la construction de grandes maisons et de kivas (généralement circulaires), la constitution de villages et de villes ${ }^{23}$, ainsi que par l'exécution de peintures rupestres. Dans une deuxième période (1100-1300) sont édifiées, à Mesa Verde au nord et à Chaco Canyon à l'ouest, des maisons-falaises et des places-fortes défensives en même temps que 


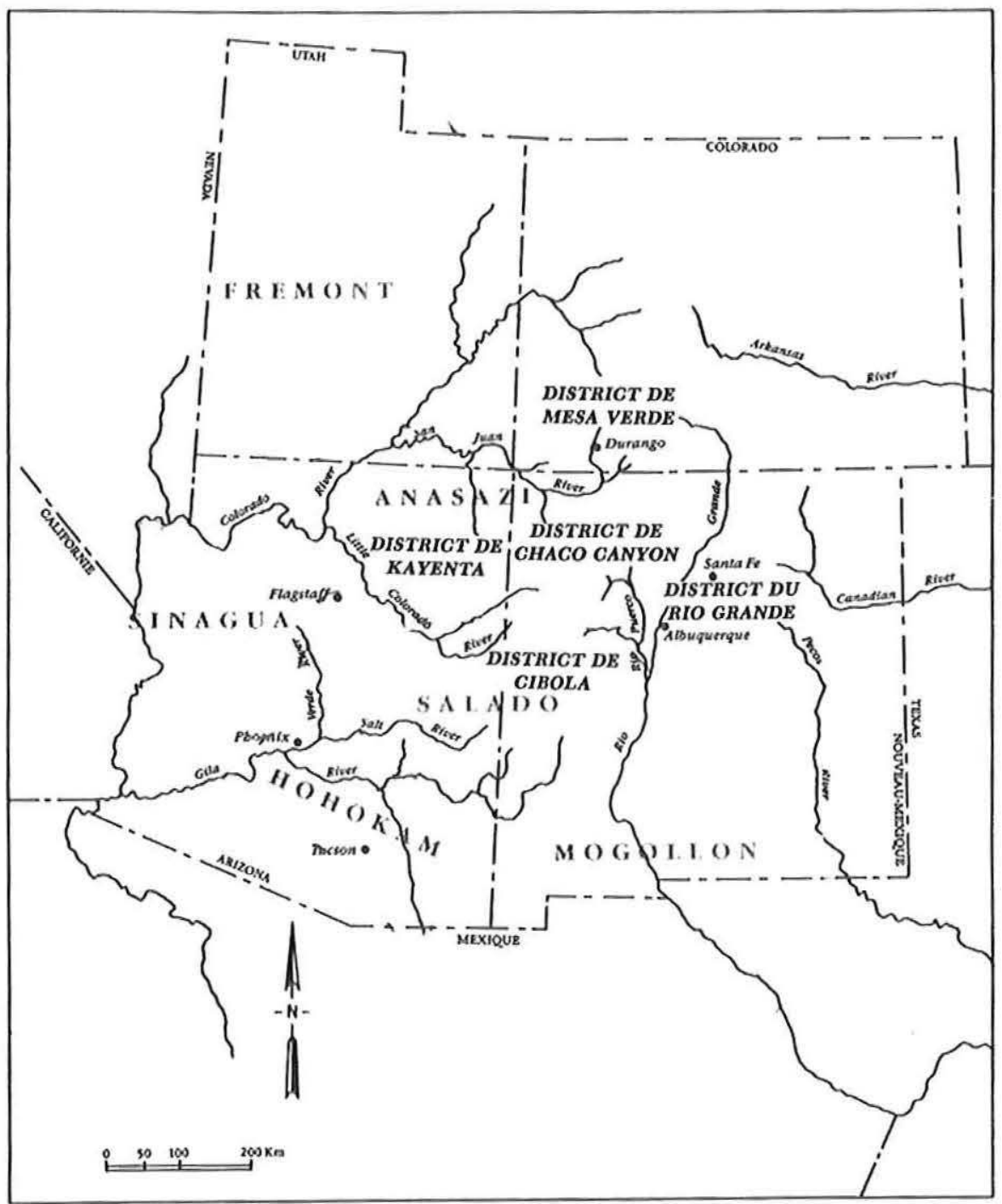

FIG. 3 - Aires culturelles du Sud-Ouest (d'après Brody 1993, carte 4, p. 15).

sont installés des systèmes d'irrigation très élaborés tels que des barrages, des réservoirs et des canaux.

Vers la fin du XIII ${ }^{\mathrm{e}}$ siècle culminent de grands bouleversements qui forcent la population à abandonner les lieux. Ils sont dus soit à des changements climatiques qui auraient provoqué une famine, soit à des incursions de groupes ennemis 
venus du nord. Peu à peu, à partir du milieu du XIII ${ }^{\mathrm{e}}$ siècle, les régions de Chaco Canyon, puis vers 1300, de Mesa Verde et de Kayenta sont abandonnées. Leurs populations se dispersent et se déplacent vers le sud et l'est ; certaines se réfugient chez les Zuni qui ont à peu près le même modèle culturel ${ }^{24}$.

De nouvelles populations venues des Plaines s'installent sur les territoires anasazis abandonnés. Elles sont nomades, parlent des langues appartenant au tronc athapaskan originaire du Nord-Ouest comme l'apache, le navajo, le shoshoni et le havasupai.

Ce sont les résultats de fouilles archéologiques pratiquées dans des sites de ces trois régions septentrionales qui ont apporté des informations intéressantes sur des rituels probablement sacrificiels ${ }^{25}$. Ces informations sont dues, en particulier, au travail des bio-anthropologues sur du matériel osseux humain découvert dans les fouilles.

Les évidences archéologiques ne datent pas toutes de la même époque. Dans le premier cas, celui de la Vallée de Kayenta, elles appartiennent à la période Basketmaker III (500-750), immédiatement antérieure à la période Pueblo I (750-900), date acceptée du début de la culture anasazi. Dans les autres cas, les évidences appartiennent à la période Pueblo I.

\section{La Vallée de Kayenta}

Au cours de fouilles dans différents sites de cette vallée, une tête trophée, avec sa peau et ses cheveux, bien conservée grâce à la sécheresse du climat, a été découverte dans une grotte (Kidder et Guernsey 1919, pp. 190-191; Guernsey 1931) ${ }^{26}$. La tête, datée de la période Basketmaker III, avait été disposée sous le corps naturellement momifié d'une jeune femme, au niveau des épaules, à proximité des restes d'un enfant et de son berceau. Il s'agit probablement de la mère. Le visage a été reconstitué à partir du scalp complet qui avait été détaché des os du crâne en trois fragments. Le premier était constitué du cuir chevelu (dos de la tête jusque derrière les oreilles); le deuxième, de la peau de la partie supérieure du visage (du haut du front jusqu'à la lèvre supérieure) ; le troisième, de la peau de la partie inférieure du visage (de la lèvre inférieure au menton et au bas des joues).

Après avoir été probablement tannées, les trois parties ont été cousues ensemble au moyen d'une couture horizontale sur le front au ras du cuir chevelu, d'une couture verticale de chaque côté devant les oreilles et d'une couture horizontale pour unir le haut et le bas du visage au niveau de la bouche. Sourcils et cils ont été conservés; nez et cavités oculaires, bien que réduits, sont reconnaissables. On note la présence de fibres de yucca dans des trous pratiqués dans les oreilles qui devaient être ornées.

La peau porte des traces de peinture faciale. Une couche d'un pigment verdâtre la recouvre complètement. Une fine raie horizontale rouge a été dessinée en haut du front, au-dessous de la couture supérieure ; le bas du visage (du bas des 
yeux au menton) a été peint en blanc; une fine raie médiane jaune partage la chevelure. Deux perforations symétriques ont été pratiquées dans le cuir chevelu pour permettre le passage d'une lanière et pendre la tête, tel un médaillon, comme cela semble avoir été souvent l'usage parmi les groupes américains qui pratiquaient le culte de la tête-trophée. La coiffure se compose de deux « couettes » et d'une natte dans le dos. Elle rappelle, selon Kidder et Guernsey (1919), les coiffures des femmes indiennes des Plaines.

Une tête-trophée assez semblable, datant de la même époque, a été trouvée, au sud-est de l'Utah, dans le site de Grand Gulch ${ }^{27}$, à l'intérieur d'une maisonfalaise. Elle reposait à proximité de squelettes masculins. La présence de propulseurs et de nombreux sacs confectionnés en peau tannée de petits animaux - cervidés ou chiens - semble témoigner à la fois de la pratique de la chasse et de la guerre, pendant la période de Basketmaker III.

Toujours au sud-est de l'Utah, ont été découverts, dans le site Cave $7^{28}$, les restes de 92 individus dont les os du crâne, certaines côtes et vertèbres portent des marques de fractures intentionnelles. Les traces laissées sur les crânes suggèrent que le scalp, oreilles comprises, était prélevé. Par ailleurs, certaines têtes ont été séparées des corps pour probablement en faire des trophées.

\section{Chaco Canyon}

Au nord-ouest du Nouveau Mexique, en 1926, Frank Roberts (1993) découvrit, dans un site daté de 900 de notre ère (Pueblo I), un charnier dans une " pit-house » (maison semi-souterraine). Il est constitué de huit squelettes incomplets d'hommes et d'adolescents qui montrent des traces de violence : fractures crâniennes, coupures, abrasions et brûlures. De nombreuses vertèbres ont disparu, probablement brisées pour en extraire la moelle. Après avoir consommé les corps, les auteurs de ces actions ont quitté le lieu, abandonnant dans le désordre les restes humains ainsi que des outils éparpillés sur le sol.

D'autres découvertes importantes ont eu lieu depuis. Elles confirment non seulement la pratique de la tête-trophée, mais témoignent aussi, en particulier au nord du Sud-Ouest, de celle de l'anthropophagie.

\section{Mesa Verde}

Dans cette région située au sud-ouest du Colorado, à la fin des années 1990, des bio-anthropologues ${ }^{29}$ ont conclu, après analyse de restes humains provenant de plus de quarante sites anasazi, que des pratiques anthropophagiques ont eu cours dans la plupart de ces lieux. Ils constatent la présence sur les ossements d'entailles causées par l'emploi de couteaux, non seulement pour séparer la chair des os, mais aussi pour briser les articulations. Ils identifient des traces de " cuisson ", les os ayant été soit bouillis, soit rôtis, plutôt qu'incinérés. 
À la même époque, un complexe, similaire à celui qu'avait trouvé en 1926 Roberts, est découvert, toujours dans la région de Mesa Verde, dans le site de Cowboy Wash (Turner 1993). Les restes, éparpillés sur le sol de deux maisons mitoyennes anasazi, se composent de sept individus, dont les os démembrés et décharnés montrent des traces de découpe (là où les muscles sont détachés), de percussion (des coups ont été portés pour disjoindre les membres) et aussi de cuisson (des morceaux d'au moins trois individus ont été rôtis). Des résidus de sang humain apparaissent sur les outils utilisés pour le dépeçage. L'ensemble est daté de 1150, début de la période Pueblo III. En outre, les os sont associés ici à un coprolithe. L'équipe qui en réalisa l'analyse put conclure qu'il contenait de la myoglobine, protéine constitutive du pigment rouge du tissu musculaire humain. C'est là la preuve directe de l'anthropophagie.

\section{Correspondances avec le rituel sacrificiel mésoaméricain}

Dans un contexte de populations sédentaires agricoles, qui ont bâti villages et villes, apparaissent donc des têtes-trophées, dont certaines ont été écorchées, puis reconstituées. La tête-trophée est également présente en Mésoamérique où elle est partie intégrante du rituel sacrificiel : on l'accroche au tzompantli, on danse avec elle à la fête du tlacaxipehualiztli lors de la danse appelée motzontecomaitotia. On l'écorche aussi en trois parties qui semblent être tannées. Puis on coud les fragments entre eux (comme pour la tête scalpée de la grotte I de la vallée de Kayenta en Arizona), ce que l'on peut voir dans les représentations de plusieurs manuscrits ainsi que dans des bijoux à l'effigie de Xipe chez les Zapotèques et dans un masque en cuivre tarasque. Bernal Díaz del Castillo (1968, vol. I, p. 452) vit plusieurs de ces têtes-trophées bien tannées ( " como cuero de guantes »), non seulement celles qui avaient appartenu à d'infortunés compagnons espagnols, mais aussi celles de quelques-uns de leurs chevaux. Il décrit aussi un sacrifice de 66 soldats espagnols aux dieux Huitzilopochtli et Tezcatlipoca, avant la chute de Tenochtitlan. Au cours de cette cérémonie, le cœur des victimes fut arraché, la peau du visage écorchée avec barbe et cheveux, le corps dépecé, la chair consommée et les entrailles données aux animaux du zoo du dernier tlatoani, Cuauhtemoc (ibid., vol. II, pp. 46-47) ${ }^{30}$.

Comme en Mésoamérique, l'anthropophagie est présente dans le Sud-Ouest nord-américain. Elle semble avoir été plus pratiquée au nord des territoires anasazi et, vers 1100 , époque marquée par une grande sécheresse, ainsi que par des troubles d'ordre politique. En Mésoamérique, le nombre de sacrifices humains suivis d'anthropophagie augmentait, comme on le sait, en période de troubles, qu'il s'agisse de famines ou de guerres. Dans ce dernier cas, les sacrifices semblent avoir été utilisés, à l'instar des Anasazi, comme arme politique.

Les interprétations des chercheurs nord-américains au sujet de l'anthropophagie divergent. Selon le courant représenté par C. et J. Turner (1999), elle 
s'inscrivait, dans le Sud-Ouest, dans le cadre d'un culte qui aurait pu être introduit depuis la Mésoamérique. Selon d'autres chercheurs, elle n'aurait été que circonstancielle. L'anthropophagie aurait surgi soit lors d'une période de famine provoquée par la sécheresse (vers 1100, selon White 1992), soit lors de graves troubles politiques qui eurent lieu vers 1150, fin de l'ère Chaco, selon J. Kantner (1999) ${ }^{31}$. En fait, peu de spécialistes, à l'exception des Turner, estimait qu'il s'agissait d'anthropophagie rituelle.

\section{Groupes du Texas}

Le territoire du Texas est intéressant parce que s'y sont établies des populations aussi diverses que leurs habitats (Newcomb 1984, pp. 21-25) ${ }^{32}$. Au moment de la Conquête, quatre aires culturelles jouxtaient ses frontières : au sud, la Mésoamérique ; à l'ouest, le Sud-Ouest ; au centre, les Plaines ; à l'est, les Eastern Woodlands.

Nous distinguerons ici deux groupes, celui des sédentaires et celui des nomades, en ne retenant parmi eux que les populations susceptibles d'avoir pratiqué des rituels sacrificiels, peut-être proches des rituels mésoaméricains.

\section{Les populations sédentaires}

Il s'agit fondamentalement des Caddo, des Wichita et des Apaches Lipan.

\section{Les Caddo}

Appelés aussi Hasinais, ils constituent le groupe le plus important et le plus ancien, celui qui avait atteint la plus grande complexité culturelle au Texas ${ }^{33}$ où ils occupaient un territoire très étendu à l'est de l'État (Figure 4). Les Caddo étaient les héritiers d'une culture de bâtisseurs de centres cérémoniels spectaculaires (buttes artificielles rectangulaires à sommet plat surmontées d'un temple ou d'un palais) organisés autour d'une place centrale et entourés de maisons. Cette culture, considérée comme étant apparue vers 500 de notre ère, était encore pleine de vigueur au XIII ${ }^{\mathrm{e}}$ siècle $^{34}$. À l'époque de l'arrivée des Européens, les Caddo ne vivaient plus que dans des hameaux, mais ils avaient conservé de leur important passé le même territoire, ainsi qu'une organisation politique élaborée qui était une sorte de confédération. Malgré leur filiation linguistique avec le Nord et l'Ouest ${ }^{35}$, ils partageaient au $\mathrm{XvI}^{\mathrm{e}}$ siècle des traits culturels avec d'autres populations de l'Est, établies dans la vallée inférieure du Mississippi. Creek, Chickasaw, Choctaw, Cherokee et, surtout, Natchez (considérés comme les parents pauvres des Caddo) comptaient parmi elles ${ }^{36}$. Avec leurs voisins de l'ouest, les Anasazi, ils troquaient arcs, bois et sel contre des couvertures en coton et des turquoises (Newcomb 1984, p. 299). Ils habitaient dans les plaines chaudes et humides et la 


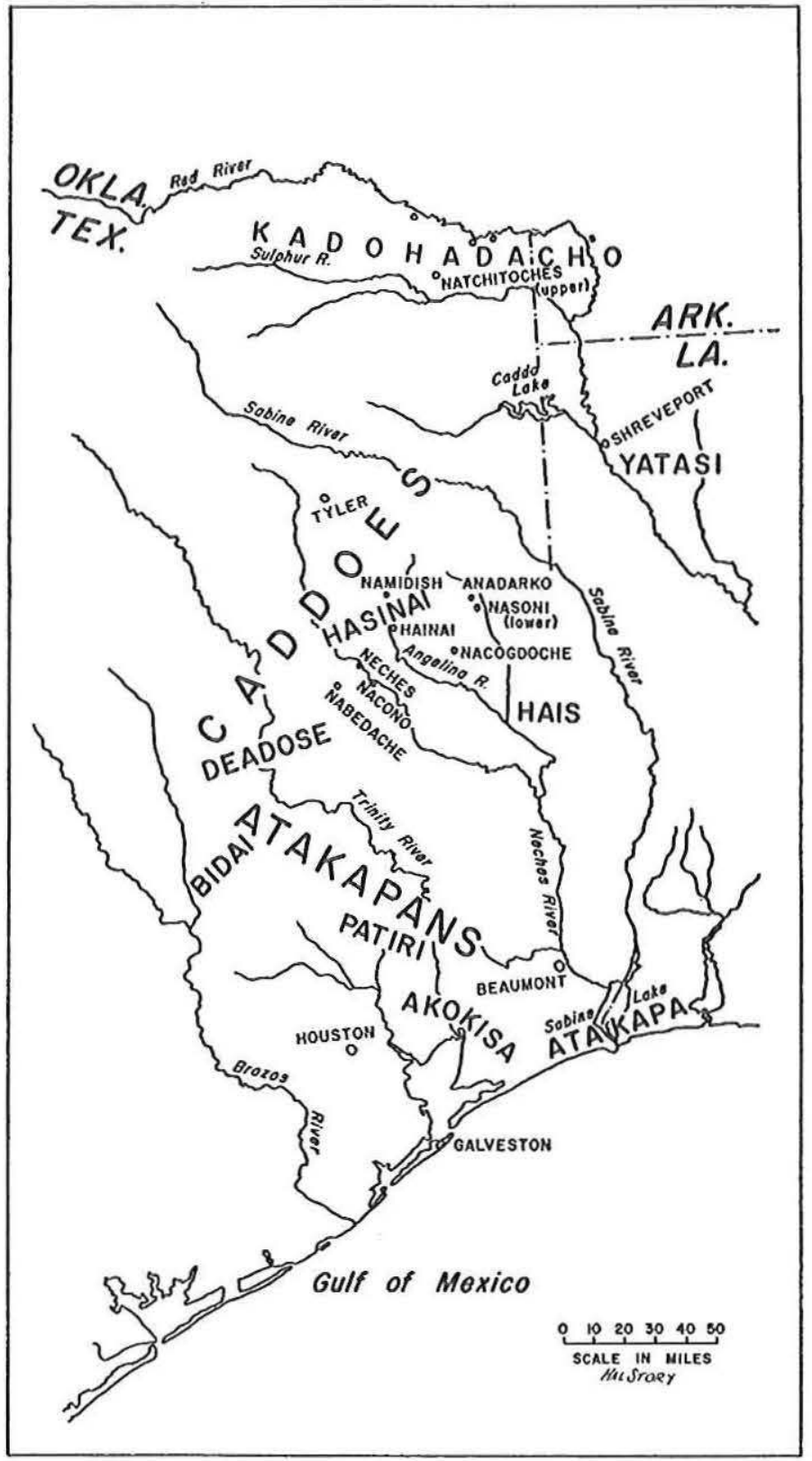

FIG. 4 - Aires du Texas appartenant à la tradition culturelle du Sud-Est (d'après Newcomb 1984, carte 4, p. 281). 
forêt ; leur territoire était riche, les terres fertiles, le gibier abondant (ours brun, cerf, bison) et les rivières poissonneuses. En plus de la chasse, ils pratiquaient la cueillette ${ }^{37}$ et l'agriculture. Dans des jardins communautaires poussaient le maïs, les haricots, les courges, le tournesol et le tabac. Les Caddo étaient connus pour leurs poteries et le tannage des peaux de cervidés et de bisons ${ }^{38}$.

L'état de guerre était constant avec leurs ennemis traditionnels, Wichita et Osage (Figure 5), ainsi qu'avec les Apaches, devenus leurs ennemis après l'arrivée des Européens (ibid., pp. 306-307, pp. 462-463) ${ }^{39}$. L'expédition guerrière pour se procurer un sacrifié obéissait à deux motifs principaux : venger les proches tués ou quête de gloire personnelle. Elle était minutieusement préparée pendant la semaine qui précédait l'action et son chef était élu en assemblée restreinte. Les guerriers qui y participaient étaient isolés dans une maison spécialement construite à cet effet avant le départ (elle était détruite après) et ils exécutaient un rituel complexe de jeûne, d'offrandes, de danses, de prières et probablement d'abstinence sexuelle. À la fin de l'expédition, les guerriers victorieux prenaient des scalps sur place, en écorchant une grande surface de la peau de la tête et du cuir chevelu (les scalps étaient plus grands que ceux des Plaines). Les prisonniers, dont parfois des femmes et des enfants, étaient tués, souvent après avoir été torturés. Certains captifs de sexe masculin étaient amenés au village des vainqueurs et livrés à la vindicte des femmes. Elles leur coupaient les doigts ainsi que de petits morceaux de chair (que d'autres captifs étaient forcés de manger) et énucléaient leurs yeux, avant de les achever.

Il y avait chez les Caddo un rituel sacrificiel offert au soleil qui ressemblait au tlacacaliliztli (ibid., p. 309). Trois jours durant, les victimes étaient pendues à des barres horizontales par les bras. Les pieds, qui ne touchaient pas le sol, étaient attachés par des lanières à un pieu planté devant les victimes de façon à maintenir leur corps sous tension. Le premier jour, les victimes étaient attachées le matin face au soleil levant, puis détachées et à nouveau attachées, le soir, face au soleil couchant. Elles ne recevaient aucune nourriture ce jour-là. Le lendemain matin, les victimes étaient à nouveau attachées. Quatre vieux officiants pratiquaient des incisions sur leurs membres supérieurs et inférieurs et recueillaient le sang qui s'écoulait des blessures. Ils le passaient à deux autres officiants qui le faisaient cuire et le donnaient à manger aux femmes et aux enfants. Les victimes, une fois mortes, étaient dépecées, cuites et consommées par les gens du village.

\section{Les Natchez}

Autre groupe de la vallée inférieure du Mississippi, les Natchez (Newcomb 1984, p. 308 ; Linton 1926, pp. 462-463) ${ }^{40}$, descendants plus ou moins déchus des " mound-builders », pratiquaient sur leurs captifs de guerre un rituel sacrificiel qui rappelait, en partie, celui du tlacacaliliztli. 


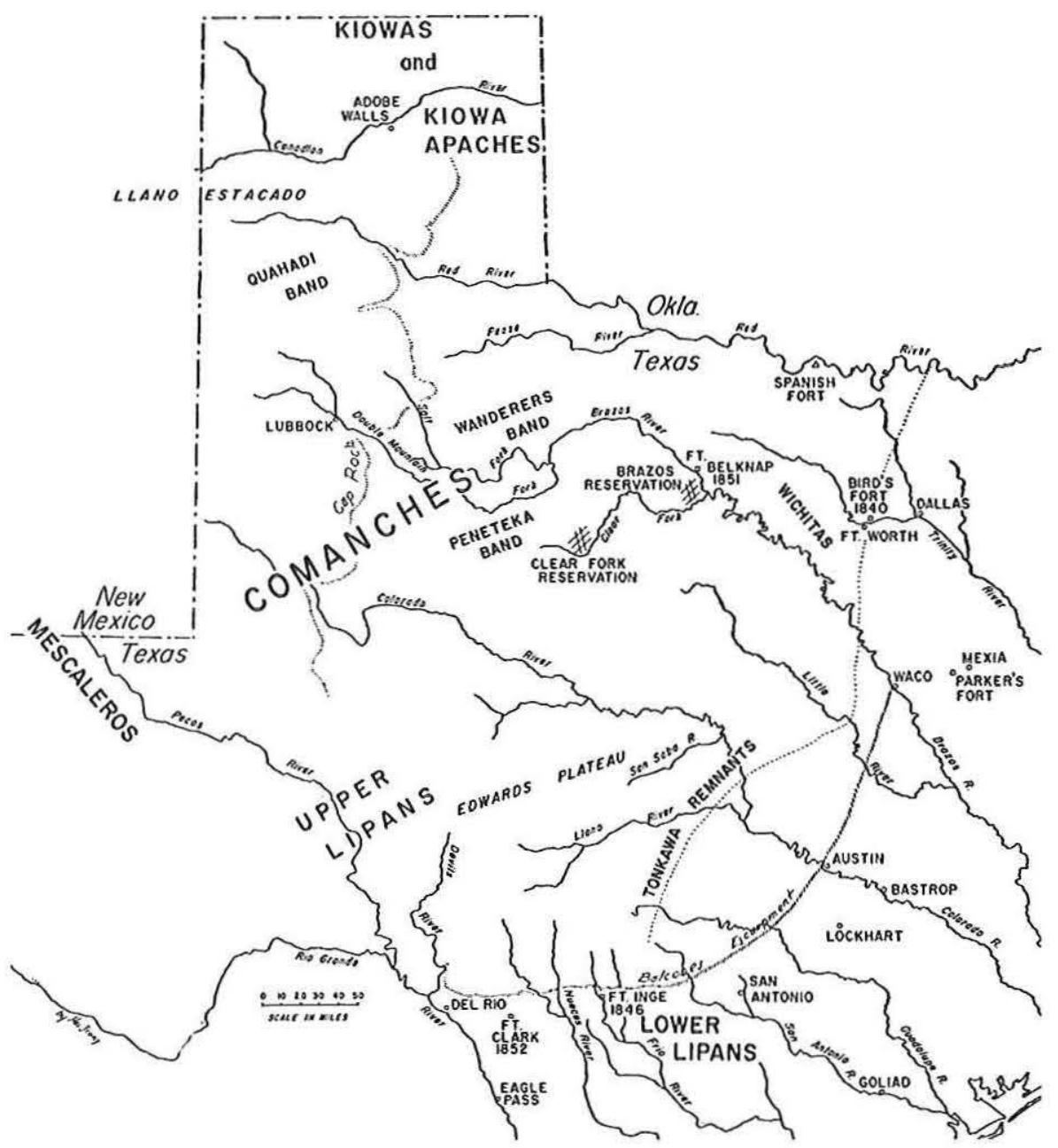

FIG. 5. - Groupes du Texas appartenant à la tradition culturelle des Plaines au XIX ${ }^{e}$ siècle (d'après Newcomb 1984, carte 3, p. 161).

Nous disposons du récit d'un planteur français ${ }^{41}$ installé en territoire Natchez au début du XvIII ${ }^{\mathrm{e}}$ siècle, témoin de la mise à mort d'un prisonnier de guerre. Gardé par de vieux guerriers, le prisonnier était attaché au bas d'un chevalet constitué de deux poteaux ${ }^{42}$ verticaux et de six horizontaux qui formaient une sorte de croix. Il était sommé de chanter jusqu’à la fin du sacrifice et pouvait, s'il le désirait, consommer un dernier repas (avant de recevoir les premiers coups). On 
le faisait avancer et se montrer aux habitants du village. Puis, celui qui l'avait capturé l'assommait d'un coup de gourdin derrière la tête, en poussant un cri de mort, et le scalpait avec le plus grand soin, sans déchirer la peau. Aux poignets du prisonnier étaient attachées des cordes dont les extrémités libres étaient envoyées par-dessus la partie supérieure du chevalet. Les cordes étaient alors tirées depuis l'arrière du chevalet afin de hisser le corps vers le haut. Les pieds étaient placés sur le poteau du bas, les chevilles attachées aux angles inférieurs et les mains aux angles supérieurs du chevalet, les quatre membres formant ainsi une croix de Saint André. Celui qui avait capturé le prisonnier brûlait, avec un tison ardent, le bras avec lequel ce dernier s'était défendu. Tous, l'un après l'autre, s'acharnaient sur la victime jusqu'à ce que mort s'ensuive.

\section{Les Wichita}

Ce groupe ${ }^{43}$, le plus septentrional du Texas, parlait l'une des quatre langues de la famille linguistique Caddo. Les Wichita, originaires du Sud de cet État, auraient émigré au nord, puis seraient retournés dans le Sud aux XVII ${ }^{\mathrm{c}}$ et XVIII ${ }^{\mathrm{e}}$ siècles, poussés par les Osages et les Comanches. Bons chasseurs et bons agriculteurs, ils vivaient dans des villages souvent fortifiés et, en accord avec la tradition des Plaines, étaient de grands guerriers (Newcomb 1984, pp. 261-277). Organiser des expéditions guerrières pour en rapporter des scalps, des prisonniers et, à partir du XvII ${ }^{\mathrm{e}}$ siècle, des chevaux, était dans leur société l'unique moyen d'obtenir du prestige.

\section{Les Apaches Lipan}

Ce groupe avait probablement été aux $\mathrm{XVI}^{\mathrm{e}}$ et $\mathrm{XVII}^{\mathrm{e}}$ siècles le plus puissant des Plaines méridionales (Newcomb 1984, pp. 103-131). Venus du nord à une époque encore inconnue, les Apaches parlaient une langue appartenant au groupe athapascan du Nord-Ouest. Ils formaient une population sédentaire d'agriculteurs, de chasseurs et, de par leur origine d'Indiens des Plaines, de guerriers qui avaient pour ennemis les Comanches. Ils prenaient des scalps, dépeçaient leurs ennemis morts et en donnaient des morceaux à manger à leurs guerriers les plus valeureux. Ils faisaient aussi des prisonniers qu'ils adoptaient ou qu'ils torturaient en leur coupant des petits morceaux de chair ou en les brûlant lentement. Ils pratiquaient la danse du scalp (voir infra Figures 6 et 7).

\section{Correspondances avec les rituels sacrificiels mésoaméricains}

Elles sont perceptibles surtout chez les Caddo et, à un degré moindre, chez les Natchez et les Wichita. Ces groupes sont des sociétés sédentaires d'agriculteurs spécialisés, mais pour qui la chasse demeure une activité importante et chez qui la guerre est endémique. 
Les correspondances sont nombreuses :

On observe en particulier que, chez les Caddo, les rituels guerriers se mêlent aux rituels sacrificiels et, probablement, aux rituels agraires : ces derniers célébraient les premières récoltes, comme dans le sacrifice à Xipe Totec où étaient consacrés les premiers fruits de la terre (Newcomb 1984, p. 313). Comme chez les Mexica, la préparation ainsi que le retour des expéditions guerrières prend une forte connotation religieuse : offrandes aux divinités (le soleil chez les Caddo), observation de périodes de jeûne, de veille et d'isolement (souvent) dans un bâtiment réservé uniquement aux guerriers (tel le Cuauhcalli dans l'enceinte sacrée de Tenochtitlan). Les groupes nord-américains concernés observaient probablement aussi une période d'abstinence sexuelle comme en Mésoamérique. Dans les deux aires culturelles, le sacrifié est un prisonnier de guerre. Le trophée de guerre est montré en signe de bravoure par celui qui a capturé le prisonnier. Il est toujours constitué par une partie du corps de ce dernier. Chez les Wichita et chez les Caddo (ibid., p. 307), c'est son scalp tanné qui est soit exposé devant la porte de la maison du vainqueur; soit porté par lui en sautoir. Chez les Mexica, c'est le fémur pendu soit à l'entrée de la maison de celui qui l'a capturé, soit à un mât. Chez les Caddo et les Natchez, il y a mise à mort d'un prisonnier attaché à un chevalet qui ressemble à celui utilisé lors du tlacacaliliztli mésoaméricain. Comme chez les Mexica, les Mixtèques et les Cuicatèques, la mise à mort est collective puisque nombreux sont ceux qui y participent. Chez les Caddo, le sang des blessures du sacrifié est récupéré par des vieillards qui font, comme dans le rituel du tlacaxipehualiztli, un peu office de cuacuacuiltin. Chez les Mixtèques et Cuicatèques, le sang est absorbé par la terre dans le rituel du tlacacaliliztli ; chez les Caddo, il est donné, cuit, aux femmes et aux enfants afin qu'ils le consomment.

La prise de scalp, le dépècement du corps, la cuisson de la chair et l'anthropophagie qui suit, apparaissent partout au Texas parmi les populations sédentaires, à l'exception des Wichita qui ne pratiquaient pas l'anthropophagie.

\section{Les populations nomades}

Il s'agit de trois groupes: les Tonkawa, les Karankawa et les Atakapan (Figure 6).

\section{Les Tonkawa}

Groupe le plus représentatif des Indiens des Plaines du Sud, les Tonkawa occupaient le plateau Edward au centre du Texas (Newcomb 1984, pp. 133153) ${ }^{44}$. Ils ne connaissaient pas l'agriculture et vivaient de la chasse aux grands mammifères - bisons, cervidés -, de la cueillette - noix de pécan - et de la pêche, dans des campements de tipis en peaux de bison. Parmi les traits propres à la culture des Plaines, ils avaient l'usage du travois tiré par des chiens, s'habillaient 


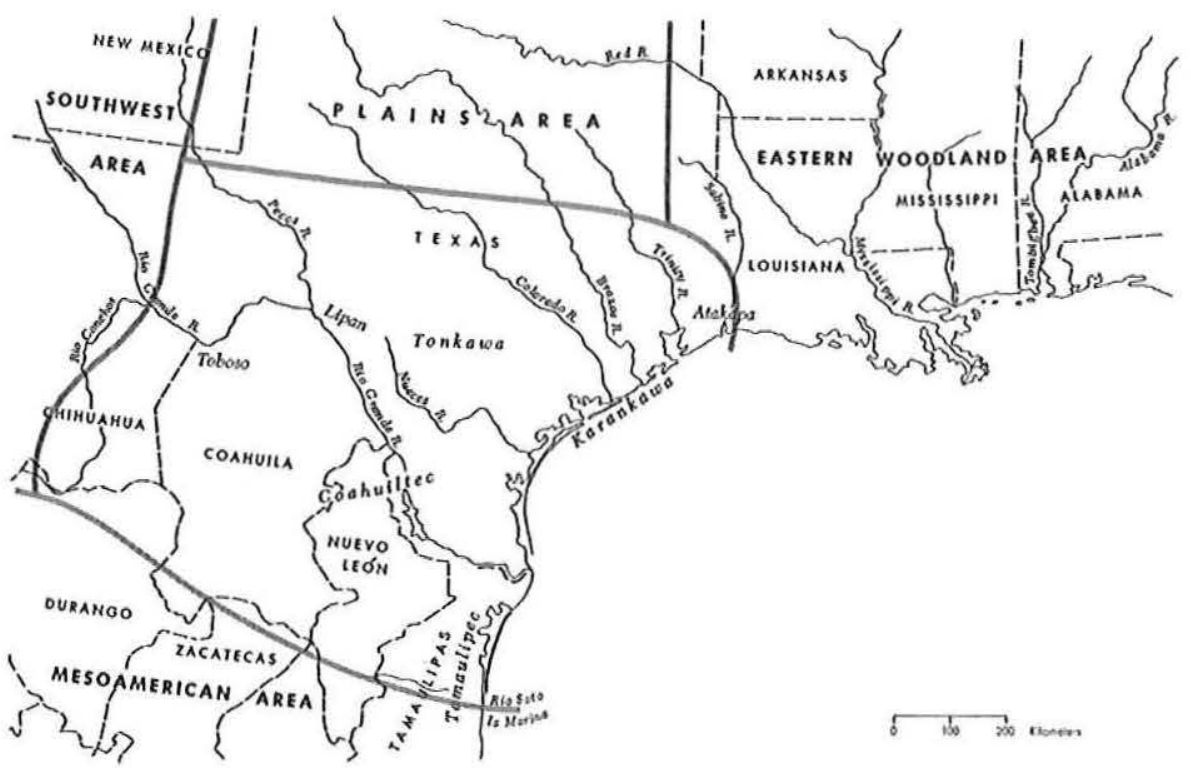

FIG. 6 - Groupes du Texas et de la Vallée inférieure du Mississippi (d'après Willey 1966, p. 331).

avec des vêtements en peaux de bison et portaient la coiffure composée soit de cheveux tressés avec des poils de castor, soit d'une couronne de plumes.

Les Tonkawa pratiquaient l'anthropophagie rituelle sur leurs ennemis. Nous en retiendrons deux témoignages directs donnés par des Occidentaux présents au moment des faits, pendant la première moitié du XIx ${ }^{\mathrm{e}}$ siècle. Dans le premier témoignage ${ }^{45}$, il s'agit du sort réservé au cadavre d'un de leurs ennemis jurés, un Comanche. Il est scalpé, sa chair détachée des os est mise à cuire dans une marmite avec du maïs et des pommes de terre ; tout le monde en mange. Dans le deuxième témoignage, il s'agit, cette fois, du cadavre d'un Wichita (Jenkins 1958, pp. 77-78). Il est scalpé, ses jambes sont coupées aux genoux, les mains aux poignets, les ongles des mains et des pieds sont arrachés. Ils ont fait un collier de ces ongles qu'ils portent autour du cou. Des flèches sont ensuite décochées dans la tête du sacrifié jusqu'à ce qu'elle soit mise en pièces, action censée apporter de la chance. Les Tonkawa empruntent au témoin occidental présent à leurs côtés un morceau de bœuf pour le faire cuire avec les pieds et les mains du mort. Ils érigent un mât sur lequel ils attachent le scalp, les mains et les pieds du sacrifié. Puis commence une danse autour du mât, qui peut durer de trois à cinq jours et au cours de laquelle les femmes mordillent de temps en temps dans les mains et les pieds cuits du sacrifié. L'ingestion des morceaux de chair des mains et des pieds par les femmes était censée faire des enfants qu'elles porteraient des êtres coura- 
geux qui härraient leurs ennemis et seraient capables d'affironter les dangers et la dureté de la vie.

Selon Newcomb (1984, pp. 149-152) ${ }^{46}$, il n'est pas étonnant que les Tonkawa aient pratiqué l'anthropophagie rituelle, étant donné l'importance du monde des morts dans leurs croyances. L'ingestion d'une partie du corps de l'ennemi entrầnait l'un des deux (ou les deux) effets suivants : en premier lieu, celui qui consommait un morceau de l'ennemi pouvait acquérir une partie ou l'ensemble du pouvoir de son âme. Si l'ennemi avait été courageux, celui qui le mangeait acquérait son courage, sa bravoure. En deuxième lieu, consommer le corps d'un ennemi était considéré comme un moyen d'insulter et d'avilir non seulement les âmes des ennemis (morts), mais aussi les ennemis vivants. L'on pouvait attendre des âmes des morts qu'elles manifestent leur courroux auprès de leurs compagnons d'armes, pour ne pas avoir été assez valeureux pour réussir à empêcher leur propre mort. L'action de manger un ennemi était censée détruire complètement son âme et, de cette façon, pour un Tonkawa, assurer la plus grande des victoires.

\section{Les Karankawa}

Ils étaient établis sur le littoral du Golfe du Mexique ${ }^{47}$ où ils menaient une existence de nomades maritimes (Figure 6). Grands nageurs, bons pêcheurs et marins, les Karankawa chassaient aussi le cerf, éventuellement le bison, l'antilope et l'ours. Fondée sur les seuls produits de la chasse, de la pêche et de la cueillette, leur existence était assez précaire, comme en témoignent des récits d'Européens ${ }^{48}$.

Les Karankawa semblent ne pas avoir été particulièrement belliqueux avant l'arrivée des Européens. Nous disposons cependant d'un témoignage de seconde main sur un sacrifice humain raconté au XVIII ${ }^{\mathrm{e}}$ siècle par Solis (1931, pp. 42-43). Un captif est attaché à un poteau, la foule danse autour de lui, armée de couteaux. Tous s'approchent de la victime vivante, lui découpent un morceau de chair qu'ils vont faire rôtir et manger sous ses yeux. La victime est ainsi coupée en petits morceaux jusqu'à ce qu'elle meure. Son scalp est pris, ses os sont distribués puis rongés et leur moelle extraite est consommée. À la description de ce sacrifice suit une autre version où la victime est pendue par les pieds au-dessus d'un feu. Elle est mangée, une fois rôtie. Selon Newcomb (ibid., p. 78), la finalité de l'anthropophagie chez les Karankawa était double. Elle était d'abord une façon d'acquérir le courage de l'ennemi en consommant certaines parties de son corps. Ensuite, elle tenait lieu de suprême vengeance. C'est ce que semble bien avoir représenté la consommation de certaines parties du corps de la victime, encore vivante, par ses ennemis.

\section{Les Atakapan}

Le nom de ce groupe nomade signifie "mangeurs d'hommes » en langue Choctaw de Louisiane (Figure 6). Il habitait le territoire situé à l'est du Texas, 
entre celui des Caddo et celui des Karankawa. Les terres, inondables et marécageuses, étaient impropres à l'agriculture, mais le gibier - cerf, ours, éventuellement bison, et caîman sur la côte - y était très abondant. C'était un peuple de bons chasseurs et tanneurs, de cueilleurs de coquillages et d'habiles pêcheurs.

Les Atakapan pratiquaient sur les ennemis qu'ils avaient fait prisonniers des mises à mort ritualisées. Plusieurs récits en témoignent, dont celui d'un Français au XVIII ${ }^{\mathrm{e}}$ siècle ${ }^{49}$. Les captifs de guerre étaient décapités, écorchés, puis dépecés (Folmer 1940, p. 220, cité in Newcomb 1984, p. 327). Après un repas anthropophagique partagé entre tous, les femmes commençaient une danse d'une grande gaieté qui durait deux jours sans discontinuer, où elles brandissaient un os ou un ongle d'un ennemi tué par leurs maris ${ }^{50}$.

Les Atakapan croyaient que «les hommes qui avaient été mangés par les hommes [et ceux dont la mort avait été causée par une morsure de serpent] ne pouvaient pas commencer une deuxième vie : ils étaient éternellement damnés » (Dyer 1917). Telle était la finalité de leur anthropophagie : assurer une damnation éternelle aux ennemis qu'ils avaient capturés.

\section{Correspondances avec le rituel sacrificiel mésoaméricain}

En Mésoamérique et parmi les groupes nomades du Texas, la mise à mort et l'anthropophagie se pratiquaient toujours sur un ennemi captif de guerre. Le trophée de guerre était constitué par une partie du corps humain du sacrifié : le scalp chez les Tonkawa, le fémur chez les Mexica. Scalp ou fémur étaient attachés à un poteau ${ }^{51}$. À la fin du sacrifice avait lieu chez les Tonkawa une danse du scalp comme survenait, à la fin du tlacaxipehualiztli pratiqué chez les Mexica, la danse des têtes appelée motzontecomaitotia. Des danses avaient lieu aussi chez les Atakapan, où les participants (des femmes) brandissaient des os ou des ongles des victimes. L'écorchement était connu au moins des Atakapan. À la fin du sacrifice du tlacaxipehualiztli, éclatait la violence, en particulier sous forme de simulacres de combat. Cette violence consistait chez les Tonkawa à mettre en pièces la tête de l'ennemi pour détruire à jamais son âme et apporter en même temps de la chance à ceux qui participaient à cette destruction. Il existait une hiérarchie entre les morceaux comestibles ou non du corps humain : les doigts et les pieds chez les Tonkawa, les os ou les ongles chez les Atakapan, la cuisse chez les Mexica. La chair du prisonnier était toujours consommée cuite, mélangée à des pommes de terre au XVIII ${ }^{\mathrm{e}}$ siècle. Avant la diffusion de ce tubercule, la chair était probablement mélangée à du maïs, comme elle l'était dans le tlacatlaolli des Mexica.

\section{Les Plaines : Les Pawnee Skidi}

Ce groupe aurait émigré au XIII ${ }^{\mathrm{c}}$ siècle, depuis le Nord-Est du Texas vers le Nebraska, chassé par la sécheresse qui sévissait dans la région (Griffin-Pierce 
1995, p. 66 ; Lowie 1982). L'un des héritages de cette origine méridionale était leur langue apparentée au tronc caddoan (Figure 7) ${ }^{52}$.

Les Pawnee Skidi, semi-nomades, étaient non seulement des chasseurs renommés de bisons et de cervidés, de bons tanneurs, mais aussi des agriculteurs (maïs) ${ }^{53}$. Pendant les deux périodes de chasse - une partie de l'hiver et de l'été -, ils vivaient dans des campements provisoires de tipis portables en peaux de bison. Pendant le reste de l'année, ils logeaient dans de grandes maisons communes - qui contenaient en moyenne quarante personnes - en terre, de forme circulaire, avec un toit en dôme. Les maisons étaient groupées dans des villages permanents, protégés par des fortifications. Comme tous les groupes des Plaines, les Pawnee pratiquaient le culte des astres et vénéraient, par ordre d'importance, le soleil, l'étoile du matin, la lune, etc. Les Pawnee sacrifiaient, en été, une jeune fille à l'étoile du matin ${ }^{54}$. De ce sacrifice, minutieusement décrit par Linton (1926, pp. 457-465), nous ne présenterons ici que les traits les plus importants. S'y incarnaient la plupart des croyances du groupe et on y retrouve des détails partagés par de nombreuses populations de chasseurs des Plaines, comme la quête de vision et l'utilisation de paquets sacrés. La décision d'exécuter ce sacrifice était déclenchée par un rêve où l'étoile du matin, qui apparaissait à un homme, lui enjoignait de capturer une victime, une jeune fille d'âge approprié (Wedel 1977, p. 134). L'homme se dirigeait vers le gardien du paquet consacré à l'étoile du matin et recevait de lui le costume de guerre qui s'y trouvait rangé. Il quittait alors son village avec des volontaires et menait une expédition guerrière nocturne dans un village ennemi. Dès que la jeune fille était capturée, le groupe cessait les hostilités et s'en allait. La jeune fille était immédiatement consacrée à l'étoile du matin et confiée d'abord au chef de l'expédition, puis au chef du village. Pendant la période qui précédait le sacrifice, elle était traitée avec gentillesse et respect ${ }^{55}$, mais seuls les chefs de l'expédition et du village pouvaient la toucher. Tout homme qui transgressait cette règle était censé s'offrir en sacrifice à la place de la jeune fille et s'il mourait avant le jour de l'exécution, elle était libérée.

On ne connaît pas très bien les rituels mis en œuvre pendant les trois premiers jours de la cérémonie qui durait en tout un peu plus de quatre jours (la victime était tuée au début du cinquième). On sait seulement que tout le village chantait les exploits de l'étoile du matin et offrait de la fumée et de la viande séchée au paquet sacré qui lui était attribué. La jeune fille était, dès le début, purifiée avec de la fumée, peinte en rouge, puis vêtue d'un costume noir qui provenait du paquet sacré. Celui qui l'avait capturée revêtait un costume rangé auparavant dans le même paquet, les deux protagonistes semblant avoir personnifié respectivement, pendant toute la cérémonie, lui l'étoile du matin, elle l'étoile du soir ${ }^{56}$. Un foyer constitué de quatre bûches brûlait pendant quatre jours, les bûches se rejoignant au centre, leurs extrémités extérieures orientées vers les quatre points cardinaux.

Au coucher du soleil du quatrième jour, après avoir chassé le public du lieu de la célébration ${ }^{57}$, quatre officiants dessinaient quatre cercles sur le sol pour 


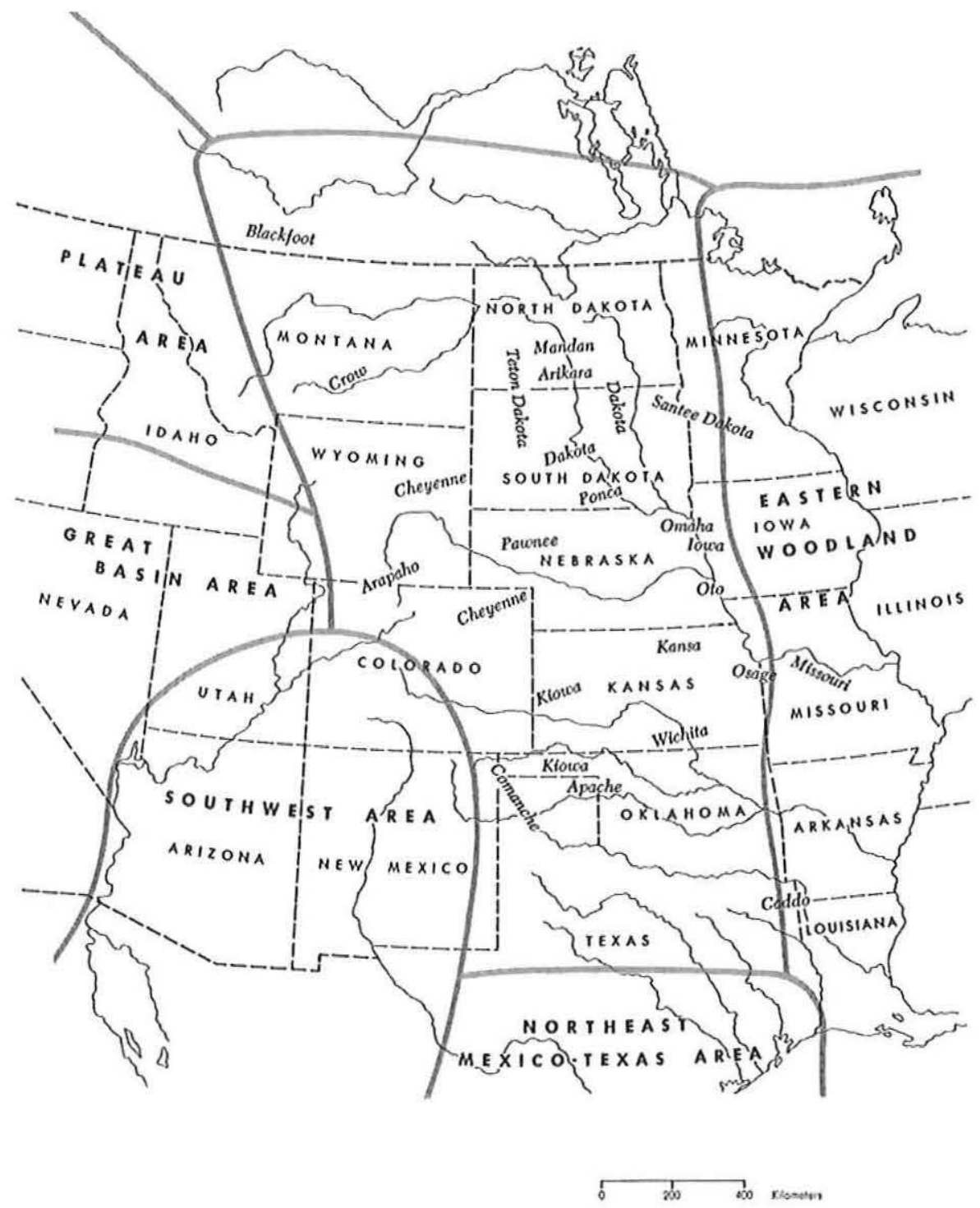

FIG. 7 - Groupes des Plaines (d'après Willey 1966, fig. 5-69, p. 314). 
chacune des quatre parties du monde. Le public était alors autorisé à revenir. Les officiants déclamaient un chant qui décrivait le voyage de l'étoile du matin en quête de l'étoile du soir pendant que l'un d'eux dansait dans la maison, un gourdin à la main et effaçait les cercles. Puis, les officiants chantaient une longue série de chants censés leur avoir été donnés par l'étoile du soir. À la fin de chaque chant, un bâton à encoches était sorti du paquet sacré de l'étoile du matin et posé sur le sol. Quand les chants cessaient, l'un des prêtres officiants déshabillait la jeune fille, peignait la moitié droite de son corps en rouge et la moitié gauche en noir, puis la rhabillait.

Tout le monde sortait alors de la maison pour rejoindre le lieu du sacrifice où se dressait une sorte de chevalet, construit le jour même, chaque étape de la construction ayant été accompagnée de cérémonies. Il était constitué de deux poteaux verticaux et de cinq horizontaux. Les deux poteaux verticaux symbolisaient la nuit et le jour, les quatre poteaux horizontaux du bas représentaient les quatre points cardinaux, le poteau horizontal du haut, le ciel. Au-dessous du chevalet, une fosse tapissée de plumes blanches symbolisait le jardin de l'étoile du soir, à l'ouest, source de toute vie animale et végétale.

Deux hommes amenaient la jeune fille de la maison au chevalet, en la tenant attachée par les poignets au moyen de lanières. Elle était tenue jusque-là dans l'ignorance de son sort et il était de bon augure qu'elle montât sur le chevalet de son plein gré. Les mêmes hommes la déshabillaient et lui attachaient les poignets à la barre supérieure et les pieds à la plus haute des barres inférieures. Les spectateurs abandonnaient le lieu car elle devait être seule au moment où l'étoile du matin apparaîtrait. À ce moment, deux hommes arrivaient de l'est avec des tisons ardents et la touchaient légèrement sous les aisselles et au bas ventre. Quatre autres la touchaient avec des bâtons de guerre.

À ce moment, celui qui avait capturé la jeune fille arrivait en courant avec un arc et une flèche sacrés, pris dans le paquet dit « du crâne ", et lui décochait une flèche en plein cœur pendant qu'un autre lui assénait un coup sur la tête avec un bâton de guerre sorti, lui, du paquet sacré de l'étoile du matin. Un officiant lui ouvrait la poitrine avec un couteau de silex et aspergeait son propre visage avec le sang qui s'écoulait, tandis que celui qui avait capturé la jeune fille en recueillait un peu pour imbiber de la viande séchée. Tous les hommes décochaient des flèches sur le corps, faisaient quatre fois le tour du chevalet et se dispersaient. Les prêtres restaient. L'un d'eux retirait les flèches du corps et en faisait quatre piles autour du chevalet. Le corps était descendu, couché sur le sol, la tête vers l'est. La viande imbibée de sang était brûlée en offrande aux dieux, au bas du chevalet. Suivaient alors des chants qui décrivaient l'ingestion du corps par différents animaux sauvages, puis sa destination finale à l'intérieur de la terre.

Selon l'interprétation qu'en donne Linton (1926, pp. 459-465), ce sacrifice était la représentation dramatique de la conquête de l'étoile du soir par celle du matin, la victoire du jour sur la nuit. Ces astres, parmi les plus importants de la 
cosmogonie pawnee, étaient considérés comme les « anges de vie », chargés de subvenir aux besoins des humains ${ }^{58}$. La victime personnifiait l'étoile du soir et celui qui l'avait capturée vivante personnifiait l'étoile du matin.

La victime n'était pas offerte à la divinité qu'elle avait incarnée, mais à celle qui l'avait conquise : l'étoile du matin (Dorsey 1904). Cette étoile avait dit aux hommes qu'elle leur avait donné les arcs et les flèches pour tuer les animaux, en précisant qu'il fallait que la flèche atteigne en premier lieu le cœur de l'animal (comme elle atteignait celui de la jeune fille). L'étoile du matin leur avait aussi donné des baguettes de bois à brûler sur lesquelles devaient être posés les animaux, sur le feu, afin que la fumée monte jusqu'aux êtres célestes, la fumée étant le véhicule de la prière des hommes.

De nombreux sacrifices étaient consacrés à cette étoile : depuis celui du premier oiseau tué à l'arc par un petit garçon, en passant par celui de grands animaux ${ }^{59}$, jusqu'aux plus importants : le scalp d'un homme ou d'une jeune fille. Avant de se servir de l'arc, qui était l'instrument de la mort, il était d'usage de prononcer le nom de l'étoile du matin. Nommer cette étoile en présence d'un animal ou d'un être humain désignait celui-ci comme étant voué au trépas.

Linton (1926) reconnaît l'existence de certains traits de ce sacrifice dans d'autres aires culturelles, en particulier chez les Zuni du Sud-Ouest et chez les Natchez du Sud-Est ${ }^{60}$. Selon cet auteur, ces deux groupes auraient reçu des influences en provenance des hautes cultures mésoaméricaines. De fait, il semble y avoir eu, depuis la Mésoamérique, deux centres de diffusion. L'un exerçait son influence sur le Sud-Est, à partir de la côte du Golfe ; l'autre sur le Sud-Ouest à partir des Hauts Plateaux. La personnification de la divinité et l'arrachement du cœur seraient arrivés chez les Pawnee depuis les Hauts Plateaux mexicains en passant par le Sud-Ouest, le chevalet et le culte aux étoiles par la vallée du Mississippi, où ils étaient parvenus depuis la Huasteca. Mais la combinaison des deux pratiques dans le rituel pawnee représente apparemment un développement local indépendant.

\section{CONCLUSION}

En dépit des différences culturelles qu'elles manifestént, les populations dont il a été question ci-dessus, sédentaires ou nomades, eurent en commun la pratique de la chasse, de l'agriculture et de la guerre, cette dernière ayant, chez les Mexica, atteint une dimension sacrée avec la xochiyaotl (" guerre des fleurs »). Toutes pratiquaient des rituels sacrificiels que l'on peut classer dans la catégorie créée et appelée par Valerio Valeri (1994) « meurtre rituel », concept plus large que celui de sacrifice. Cette forme ritualisée, codifiée et encadrée, de mise à mort d'un ennemi du groupe avait pour finalité générale de rapporter des bénéfices aux sacrificateurs et ceux-ci avaient pour commun dénominateur le maintien de 
l'harmonie entre les puissances divines et les hommes (ceux qui exécutent le rituel), condition première de la survie.

Nous pensons, à l'instar d'auteurs comme Huitzinga (1988, pp. 15-56), Dorsey (1904) ou encore Linton (1926) - cette opinion peut d'ailleurs s'articuler avec celle de Valeri-, que le rituel sacrificiel, offert soit à des entités surnaturelles divinisées comme les astres (soleil, étoile du matin, étoile du soir...), soit à des dieux, était la reproduction dramatique de faits accomplis par les divinités en des temps mythiques ${ }^{61}$. En les reproduisant, ces faits étaient réaffirmés et l'attention des divinités à nouveau dirigée vers les hommes; en même temps, le rituel rappelait aux humains les bénéfices dont ils étaient redevables aux divinités. Le rapport entre les hommes et le monde surnaturel était ainsi réactivé et les dieux, satisfaits des rites sacrificiels exécutés à leur intention, pouvaient se montrer cléments envers les hommes.

En l'honneur de qui était célébré le rituel ? En l'honneur du soleil (divinisé) chez les Caddo et Natchez, de l'étoile du matin ainsi que de l'étoile du soir chez les Pawnee, du dieu du feu chez les Zuni, des divinités agraires comme Xipe ou Chicomecoatl et du feu, comme Xiuhtecuhtli, chez les anciens Mexicains.

Quels étaient les autres bénéfices attendus? Au niveau collectif, ils avaient trait soit à la conservation du monde dans lequel vivait le groupe (garantir le mouvement des astres), soit à sa survie alimentaire (garantir la fertilité de la végétation), soit encore à la paix sur terre et dans l'au-delà (veiller à ce que le groupe ne soit pas importuné par les ennemis, vivants ou morts). Au niveau individuel, en mangeant un ennemi, il s'agissait principalement de s'approprier une qualité du sacrifié, force ou courage ${ }^{62}$; parfois, également, on pouvait rechercher une satisfaction de vengeance précise en torturant l'ennemi responsable de la mort d'un parent.

Quelles étaient les modalités du rituel, depuis la quête de la victime jusqu'à la disposition du corps après la mise à mort ? Partout, sa réalisation s'étendait sur plusieurs jours. Le sacrifié appartient à l'un des deux sexes et à un groupe ennemi du sacrificateur ; il représente souvent le dieu en l'honneur duquel il sera sacrifié ; plus rarement, comme chez les Pawnee, la victime représente le dieu qui sera sacrifié au profit d'un autre dieu. Il a été capturé soit lors d'une expédition guerrière menée expressément en groupe chez l'ennemi, soit au cours d'escarmouches avec ce dernier. Pendant la période préparatoire, le prisonnier est souvent isolé et généralement bien traité (Pawnee, Mexica, etc.). Cependant le nombre de personnes ayant le droit de s'adresser à lui est restreint. Ceux qui participent à la capture se sont soumis auparavant à des rituels de purification (isolement, abstinences d'ordre sexuel et alimentaire, prières...). Ils se soumettent à nouveau à ces mêmes rituels de purification avant la mise à mort.

Celle-ci est publique et nombreux sont ceux qui y participent, comme en Mésoamérique. Les modalités varient. L'immolation d'un individu en lui arrachant le cour (après une incision sur la poitrine) sur une pierre semble n'avoir eu 
lieu qu'en Mésoamérique et parmi certains groupes Pueblo du Sud-Ouest. Le rituel susceptible de partager le plus de traits entre la Mésoamérique et les groupes considérés est celui de la mise à mort d'une personne attachée à un chevalet ${ }^{63}$ (Caddo, Natchez et Pawnee) ou à un simple poteau (Karankawa). La victime mourait, atteinte par des flèches, la première en plein cœur, comme à la chasse (en Mésoamérique ainsi que chez les Caddo, les Tonkawa et les Pawnee). Chez ces derniers, simultanément, un coup de massue était asséné sur la nuque de la victime, et une fente pratiquée sur sa poitrine. La mise à mort était éventuellement accompagnée de tortures. Les Caddo mutilaient les doigts de la victime et prélevaient des morceaux de sa chair. Les Apaches Lipan prélevaient des morceaux de chair et brûlaient des parties du corps. La victime pouvait aussi mourir des suites des tortures infligées, comme chez les Natchez (coups de gourdin sur la nuque, brûlures, prise de scalps) et les Karankawa (découpée en morceaux de son vivant).

Le dépècement est une pratique courante chez les Anazasi, les Caddo, les Apaches, les Tonkawa et les Atakapan. Il y a généralement une hiérarchie des morceaux de la chair du sacrifié. Alors que, parmi les Mexica, le morceau de choix était la cuisse, chez les Tonkawa, c'étaient les mains et les pieds et, chez les Atakapan, certains os ou ongles.

La peau est écorchée chez les Anazasi et chez les Tonkawa, comme en Mésoamérique. Le cuir chevelu est scalpé dans toutes ces populations nordaméricaines. Les scalps sont des trophées de guerre et des objets de culte : on leur fait des offrandes de tabac et de nourriture, on chante en leur honneur. Chez les Caddo et les Natchez, on les dédie à des entités surnaturelles et on danse, autour des poteaux auxquels ils sont attachés, qu'il s'agisse de la danse du scalp en l'honneur du soleil, avant le départ d'une expédition guerrière ou, comme chez les Tonkawa, pour conclure le banquet anthropophagique. Les scalps sont accrochés soit à des mâts (à la manière des têtes accrochées au tzompantli chez les Mexica), soit à la porte de celui qui a capturé le prisonnier. Cette danse rappelle celle des têtes coupées qui avait lieu pendant la fête de tlacaxipehualiztli chez les Mexica. Le scalp est mis en évidence chez celui qui a capturé le sacrifié chez les Caddo, les Natchez et les Tonkawa tandis que, chez les Mexica, le fémur du sacrifié était exposé chez celui qui l'avait capturé. Le scalp était l'insigne de sa bravoure, ses titres et sa renommée dépendant du nombre de captifs qu'il avait fait pour le sacrifice. Mais le scalp était-il le siège du tonalli comme en Mésoamérique?

En ce qui concerne la tête, il existe des têtes-trophées chez les Anazasi et, bien sûr, chez les Mexica. Pour le sang, c'est celui des blessures, liquide précieux chez les Mexica qui en imbibaient les statues des divinités après l'exécution du sacrifice ; chez les Caddo et les Pawnee, il est également important. Les Caddo le recueillent et le consomment cuit ; chez les Pawnee, le sang de la sacrifiée s'écoule sur la viande qui est brûlée et offerte aux divinités astrales que le sang parvient ainsi à atteindre, porté par la fumée qui s'échappe de la viande. 
Les os du sacrifié semblent être rongés chez les Anasazi et les Karankawa et leur mœlle extraite et mangée ${ }^{64}$ : chez les Mexica, le fémur du sacrifié est un trophée ainsi que l'humérus (mais dans le cadre de cultes à d'autres divinités).

L'anthropophagie, toujours rituelle, était une pratique très répandue parmi les groupes que nous avons évoqués, à l'exception des Wichita. La chair est généralement consommée cuite avec du maïs comme chez les Mexica (ou, plus tard au XIX ${ }^{\mathrm{e}}$ siècle, chez les Tonkawa, avec des pommes de terre), les Anazasi, les Caddo et les Tonkawa. Elle est consommée rôtie chez les Karankawa et à demi rôtie chez les Caddo du Sud (Knowles 1985, p. 164). Le repas, de même que la fête, est toujours collectif.

Malgré leur puissance politique et guerrière et malgré la magnificence de leurs rituels sacrificiels, les Mexica (et d'autres groupes du Mexique central et méridional) semblent avoir conservé des souvenirs et, en particulier; des savoirfaire de leur origine septentrionale de chasseurs-cueilleurs. La mise à mort des victimes dans le rituel du tlacacaliliztli ressemble beaucoup à celle qui avait lieu plus au nord : la victime y était abattue comme un gibier, une flèche ou un dard envoyé en plein cœur par des guerriers-chasseurs. Le traitement du corps à la fin du rituel du tlacaxipehualiztli participait, lui, totalement d'un savoir-faire de chasseurs. L'écorchement du corps, son dépècement, ainsi que le traitement réservé à la peau en témoignent. Les Mexica coupaient et cousaient la peau humaine, comme ils l'auraient fait d'un cuir animal pour en façonner un vêtement, un masque facial et une coiffure ; tout cela un peu à la façon dont des officiants anasazi avaient reconstitué le visage découvert dans la grotte de Kinboko par Kidder et Guernsey (1919). *

* Manuscrit reçu en mai 2007, accepté pour publication en mai 2008.

\section{NoTES}

1. Voir Valeri (1994, pp. 104-105) qui adopte le concept élargi de sacrifice comme une forme de galaxie : de plus en plus mince au fur et à mesure qu'il s'éloigne de son centre solide. Pour Valeri, c'est la mise à mort ritualisée qui constitue le phénomène central du sacrifice. Quant aux bénéfices apportés, ils ont pour dénominateur commun d'assurer la survie et l'allongement de la vie du responsable du rituel et des invités qui y sont conviés.

2. Ce mois situé, d'après la plupart des sources, entre le 4 et le 21 mars s'achevait donc en gros par l'équinoxe de printemps.

3. Le mois ochpaniztli (" balayage ") situé, d'après la plupart des sources, entre le 31 août et le 19 septembre précédait l'équinoxe d'automne.

4. Izcalli (" étirement " car on étirait les enfants pour qu'ils grandissent) est une fête « fixe » qui a lieu entre le 18 janvier et le 6 février. C'est seulement tous les quatre ans qu'étaient pratiqués des sacrifices humains (par arrachement du ceur) pendant le mois izcalli, et seulement à Cuauhtitlan.

5. À l'intérieur de l'empire, il s'agit des enclaves que constituaient les royaumes de Tututepec et Metztitlan (Hidalgo), de la vallée de Puebla-Tlaxcala (Tlaxcala, Tliliuhquitepec, Huexotzinco, Cholollan-Totomihuacan, Zacatlan), de Yopitzinco (Guerrero), de Tototepec et de Teotitlan (Oaxaca). À l'extérieur de l'empire, il s'agit de l'empire tarasque. 
6. Selon l'Historia de los Mexicanos por sus Pinturas (1988, p. 15).

7. Dans le Codex Vaticano-Rios (f. 83v), le dernier Tlatoani, Motecuhzoma Xocoyotzin, est revêtu de la tenue de Xipe lors de la campagne militaire contre Toluca en l'année 9 maison (soit 1501 selon Seler 1960, II, p. 402).

8. Cette peinture corporelle indique que le personnage sera soumis au rituel sacrificiel du tlahuahuanaliztli (" action de rayer ou de griffer »).

9. Selon Motolinía (1996, pp. 189-190), " dos indios principales vestianse los cueros con los rostros cubiertos con máscaras y después de vestidos tomaban en las manos, en cada una, su canilla [les fémurs des femmes écorchées] [...] bajaban bramando por las gradas [...] bestias encarnizadas [...] ".

10. Il s'agit de l'Historia Tolteca Chichimeca et des codex Huamantla, Vaticano-Rios et TellerianoRemensis (voir Vié-Wohrer 1999, vol. II, pp. 103-107).

11. Fernández de Oviedo, l'un des premiers témoins espagnols de la région, publie en 1526 le Sumario de la Historia Natural y Moral de las Indias, puis, à partir de 1535, commence la rédaction de l'Historia... qui ne sera publiée dans sa totalité (quatre volumes) qu'au XIxe siècle (voir León Portilla 1972, pp. 57-58).

12. Ce n'est en fait qu'à la fin du xix ${ }^{e}$ siècle, avec la bataille de Wounded Knee en 1890 , que tout le territoire nord-américain passe sous l'autorité des colons d'origine européenne.

13. L'arrivée des Européens (Espagnols, Anglais et, dans une moindre mesure, Français qui étaient en "Louisiane " à la fin du XVII ${ }^{e}$ siècle) provoqua de profonds bouleversements parmi les Indiens : guerres, déportations, migrations pour échapper aux nouveaux venus, intrusions d'autres groupes poussés par l'avancée des Européens.

14. Edward Sapir a défini la famille uto-aztèque à la fin des années 1920 , une famille non remise en cause par la dernière classification des langues amérindiennes à la conférence de l'université d'Indiana en 1964 (Campbell et Mithun 1979, pp. 31-37).

15. Pour Valeri (1994, pp. 104-105), les sacrifices rituels apportent un bénéfice et ils visent fondamentalement l'entretien et l'allongement de la vie du responsable du rituel et de ceux qu'il y associe.

16. Il y a, de nos jours, une population de 50000 personnes distribuées en dix-neuf tribus.

17. Inintelligibles entre elles et dont certaines, à ce jour, n'ont pu être identifiées (Davis 1979, pp. 390-399).

18. Les deux groupes auraient émigré d'un foyer commun situé quelque part dans le Sud-Ouest des États-Unis (Davis 1979, p. 416).

19. Selon Yolanda Lastra de Suárez et Fernando Horcasitas (1983, pp. 263-281), il existe neuf sous-familles de ce groupe : numic (mono, panamint, comanche) ; tubatulabal ; takik (luiseno, cahuila, serrano); hopi ; pimano (papago, tepehuan); tarahumara (tarahumara, varohio); cahita (yaqui, mayo); corachol (cora, huichol); azteca (nahua).

20. À cette époque, ces produits ne fournissent qu'un complément alimentaire de ceux obtenus par la chasse et la cueillette.

21. La culture des haricots s'ajoute aux produits déjà cultivés (Brody 1993, p. 73).

22. Toujours selon Brody (1993, p. 42), les Anasazi reçurent « du Nord, de l'Ouest et du Centre du Mexique le coton, le métier à tisser, la poterie, l'irrigation et d'autres techniques agricoles, l'arc et la flèche... ". Il y avait des tissus anasazi dans des sites du Sonora ( $650 \mathrm{~km}$ au sud des Four Corners). Entre le $\mathrm{XI}^{\mathrm{e}}$ et le $\mathrm{xIV}^{\mathrm{e}}$ siècles, ils ont commercé avec Casas Grandes (Chihuahua), site qui avait lui-même des contacts plus au sud (des clochettes de cuivre de l'Occident ont été trouvées au Nouveau Mexique).

23. Certaines villes ont pu compter de 15000 à 30000 habitants.

24. La suprématie anasazi se déplace et s'affaiblit avec la conquête du territoire par Juan de Oñate en 1598. La grande révolte de 1680 (Brody 1993, pp. 48, 185) l'achève.

25. Selon des propos recueillis par Bourke (1884) en 1881, à Zuni (Arizona), auprès d'un informateur âgé, tous les Pueblo, Moqui, Zuni, Acoma, Laguna, Jemez et autres pratiquaient, il y a longtemps, des sacrifices humains lors de la fête du feu au solstice d'hiver (le 22 décembre). La victime était égorgée, sa poitrine ouverte et son cœur arraché pour recevoir de bons augures (" asking good fortune»). 
26. Les restes ont été trouvés en 1915 dans la Cave I à Kinboko (House Canyon, près de Kayenta). Ils témoignent aussi sans doute d'une mortalité infantile élevée.

27. Découverte dans la tombe 16 de la grotte I (Montgomery 1894, p. 230, in Kidder 1963, p. 38).

28. Site fouillé par Richard Wetherill en 1893.

29. White (1992) ainsi que C. et J. Turner (1999) ont fouillé le site 5MTUMR-2346 à Mancos.

30. Les empereurs mexica possédaient des réserves d'animaux sauvages.

31. Elle était une arme (assez rarement utilisée) pour semer la terreur chez l'ennemi. Les actes d'anthropophagie, selon Kantner (1999), étaient isolés et ont peu duré.

32. Elles se rattachent à quatre grandes traditions culturelles : la première est celle des chasseurspêcheurs semi-nomades qui ne connaissaient pas l'agriculture ; celle-ci se situait dans la région côtière du Golfe. La deuxième tradition culturelle était celle des Plaines du Centre et du Nord de l'État, où vivaient les Tonkawa qui avaient pour voisins, au nord-ouest de leur territoire, les Apaches. À partir des $\mathrm{XVII}^{\mathrm{e}}$ et $\mathrm{XVIII^{ \textrm {e } }}$ siècles, ces groupes sont devenus de grands chasseurs de bisons, vivant dans des campements de tipis. Ils ont adopté un modèle de vie similaire à celui des Apaches Kiowa, des Kiowa, des Comanches et des Wichita, venus des plaines plus septentrionales, pour s'installer au Texas. Selon Willey (1966, p. 311), la culture des Plaines s'étend du sud de l'Alberta, du Saskatchewan et du Manitoba au Canada jusqu'au Texas. La troisième tradition culturelle était celle du Sud-Est, la plus riche et la plus développée du Texas, dont le foyer d'origine se trouvait chez les Indiens Natchez dans la vallée inférieure du Mississippi. Les Indiens Caddo qui occupaient un territoire comprenant l'Est et le Nord-Est du Texas ainsi que des portions de Louisiane, de l'Arkansas et de l'Oklahoma, étaient les représentants les plus achevés de cette tradition culturelle. La quatrième tradition est celle de la culture circum-caribéenne qui a exercé son influence sur celle du Sud-Est (Chafe 1979, pp. 213-235).

33. Selon Newcomb (1984, pp. 279-313), il s'agit des Caddo méridionaux. Les Caddo se distribuaient en douze tribus dont la plus grande était celle des Hasinai (originaire d'Oklahoma). Ces tribus parlaient la même langue avec des différences dialectales mineures (à l'exception des langues Pawnee, Wichita et Kitsai, qui étaient inintelligibles entre elles). Des Français, dont Robert Cavelier de La Salle, les fréquentèrent à la fin du xvit ${ }^{\circ}$ siècle.

34. Selon Newcomb (1984, p. 283), il s'agit du modèle dit « mississippien » propre à l'aire culturelle du Sud-Est qui s'étendait d'ouest en est depuis la rivière Trinity (Texas) jusqu'à l'Atlantique et au nord sur des centaines de miles. Le modèle mississippien avait reçu une influence circum-caribéenne. Les collines artificielles (elles mesuraient souvent $20 \mathrm{~m}$ de haut et $200 \mathrm{~m}$ de côté) avaient une fonction religieuse. Selon Willey (1966, p. 290), elles abritaient probablement des sépultures.

35. Leur(s) langue(s) a (ont) été, à une époque, parlée(s) au cœur des grandes plaines, là où vivaient les grands chasseurs, depuis le Dakota du Sud jusqu'au Nord du Texas et jusqu'à l'est, dans les " Woodlands » de l'Arkansas et de la Louisiane.

36. Par ailleurs, il est possible qu'il y ait eu des migrations des Caraïbes à la côte du Golfe.

37. Noix de pécan, châtaignes et baies entraient dans leur alimentation.

38. Les Caddo se déguisaient en cerf pour attirer le gibier (Newcomb 1984, pp. 292-294).

39. Devenus cavaliers, les Caddo faisaient des incursions en territoire apache pour chasser le bison.

40. Les Natchez avaient été en partie soumis à des influences circum-caribéennes.

41. Antoine-Simon Le Page du Pratz (cité in Linton 1926, p. 463) s'installe comme planteur de tabac près de la Nouvelle-Orléans entre 1720 et 1728. De retour à Paris, il écrit en 1758 un Mémoire sur la Louisiane et une Histoire de la Louisiane dans laquelle il décrit en particulier les Indiens Natchez.

42. La quête des poteaux se déroule comme s'il s'agissait de la quête de gibier. Ceux qui vont les chercher miment une partie de chasse (Linton 1926).

43. Les Wichita furent des alliés des Français au XVIII ${ }^{e}$ siècle (Newcomb 1984, pp. 248-277).

44. Ce sont les Français qui sont les premiers témoins de leurs pratiques, au XVII ${ }^{e}$ siècle, puis les Espagnols. La langue des Tonkawa n'a pas été réellement identifiée, peut-être appartient-elle à la famille coahuiltèque (Newcomb 1984, pp. 134-135).

45. Il est de Noah Smithwick qui vécut parmi les Comanches, près d'Austin, vers 1838 (Newcomb 1984, pp. 150, 345). 
46. Chez les Tonkawa, les âmes des morts étaient considérées comme des êtres surnaturels qui exerçaient un maximum de pouvoir sur les vivants. En particulier, si les morts n'avaient pas été correctement enterrés, leurs âmes venaient hanter les hommes responsables de cette faute (au lieu d'aller vers leur nouveau séjour à l'ouest) et mettre leur vie en danger.

47. C'est une plaine côtière qui s'étend du Golfe de Galveston à Corpus Christi, îles comprises (Newcomb 1984, pp. 59-81).

48. Les Karankawa sont connus grâce aux témoignages des survivants (parmi lesquels Cabeza de Vaca) de l'expédition de Narvaez en 1528, auxquels les Indiens portèrent secours. Les Espagnols aftamés avaient mangé certains de leurs propres compagnons sous les yeux horrifiés des Indiens. Les Karankawa tombent alors dans l'oubli jusqu'au naufrage de Cavelier de La Salle en 1685, au cour de leur territoire, où il fonde le Fort Saint Louis. Toute la garnison fut massacrée.

49. François Simars de Bellisle (1695-1763) fut oflicier à bord du bateau Maréchal d'Estées qui se perdit aux alentours de Galveston, en 1719. Il se retrouva seul et devint esclave des Atakapan, auprès desquels il vécut presque deux ans. On lui doit, entre autres, ce récit : « À leur retour, ils jetèrent l'Indien dans la prairie. L'un d'eux coupa sa tête et un autre coupa ses bras, tout en l'écorchant. Plusieurs mangèrent la graisse jaune, qui était encore crue, et ils finirent par le dévorer complètement " (in Newcomb 1984, pp. 315-328).

50. Il s'agissait surtout des femmes du groupe atakapan akokisa.

51. Appelé tlacaxipehualizquauh chez les Mexica.

52. Le tronc caddoan auquel appartiennent les langues pawnee, kichaï et caddo (Chafe 1979, pp. 213-235). « Skidi » signifie « loup » en pawnee.

53. Ils tannaient des peaux de bison, de cerf et d'élan dont ils faisaient des vêtements, des parflèches, des sacs, des bottes et, plus tard, avec l'introduction du cheval, de la sellerie (selles, harnais).

54. L'étoile du matin, l'une des principales divinités des Pawnee, leur avait donné des arcs et des flèches pour qu'ils tuent des animaux, la flèche devant atteindre en premier lieu, le cœur. En Mésoamérique aussi, Vénus est une entité divine qui décoche des flèches.

55. Mais il était interdit de lui procurer des vêtements (Linton 1926, p. 457).

56. Il y avait une divinité suprême qui siégeait au-dessus des nuages. Elle gouvernait le monde au moyen d'ordres qu'elle donnait aux divinités inférieures, célestes ou terriennes. Chez ces dernières, par ordre d'importance, il y avait sa femme, Voûte céleste, puis une déesse, Étoile du soir. Cette étoile avait non seulement eu le privilège de donner naissance au premier être humain, une fille, mais en plus elle transmettait les ordres de la divinité suprême aux hommes sur terre. C'est à cette fille de l'étoile du soir que les Pawnee Skidi offraient en sacrifice une jeune fille (Dorsey 1904, pp. 66-70). En outre, c'était l'étoile du soir qui était responsable de tous les moyens de subsistance, des produits de l'agriculture à ceux de la chasse.

57. Le lieu où se déroule la première partie du rituel sacrificiel n'est pas spécifié dans la description de Linton (1926).

58. «L'Étoile du Matin et l'Étoile du Soir sont les Anges de la vie. Ce sont elles qui fournissent tout ce qui est nécessaire à l'homme » (Rowley Moore 1939, p. 155).

59. Les aigles (à l'exception de l'aigle blanc), le cerf, l'antilope, le chat sauvage, la loutre, le bison.

60. Chez les Dakota de l'est, des prisonniers étaient attachés à des arbres ou à des poteaux et on laissait aux jeunes garçons le droit de leur décocher des flèches (Linton 1926, p. 464).

61. Cette interprétation est proche de celle de Huitzinga (1988, pp. 36-37) à propos des fêtes sacrées.

62. Comme il a été dit plus haut, les femmes tonkawa croyaient que l'ingestion des mains et des pieds d'un sacrifié feraient d'elles des mères d'enfants forts et courageux.

63. Rappelons que chaque étape de la construction du chevalet est accompagnée d'un rituel particulier.

64. La consommation des os apparât aussi dans les manuscrits pictographiques du Mexique central. 


\section{RÉFÉRENCES CITÉES}

Acosta Saignes Miguel

1950 Tlacaxipeualiztli : un complejo mesoamericano entre los caribes, Serie de Etnología, Instituto de Antropología y Geografía, Facultad de Filosofía y Letras, Universidad Central, Caracas.

BOURKE John G.

1884 The Snake Dance of the Moquis of Arizona being a narrative of a journey from Santa Fe to the villages of the Moquis Indians, cité in Linton, American Anthropologist, 28, 1926.

BRoDY Jerry J.

1993 Les Anasazis : les premiers Indiens du Sud-Ouest américain, trad. Hélène Seyrès, Edisud, Aix-en-Provence.

CAmpbell Lyle et Marianne Mithun (éds)

1979 The Languages of Native America : historical and comparative assessment, University of Texas Press, Austin.

CHAFE Wallace L.

1979 "Caddoan », in Lyle Campbell et Marianne Mithun (éds), The Languages of Native America : historical and comparative assessment, University of Texas Press, Austin, pp. 213-235.

Codex Becker I

1996 in Codex Alfonso Caso. La vida de 8-Venado, Garra de Tigre (ColombinoBecker I), Patronato Indígena, AC, Mexico.

Codex Borbonicus

1991 Ferdinand Anders, Maarten Jansen et Luis Reyes García (éds), Akademische Druck- u. Verlagsanstalt/Fondo de Cultura Económica, Graz/ Mexico.

Codex Borgia

1963 Étude d'Eduard Seler, 3 vols, Fondo de Cultura Económica, Mexico.

Codex de Huamantla

1984 in Manuscrito de los siglos xv y xvi que se conserva en la Sala de Testimonios pictógraficos de la Biblioteca nacional de antropología e historia y en la

Biblioteca estatal de Berlin, étude de Carmen Aguilera, 2 vols, Instituto Tlaxcalteca de la Cultura, Tlaxcala.

\section{Codex Fernández Leal}

1991 éd. René Acuña, Universidad Nacional Autónoma de México, Instituto de Investigaciones Filológicas, Mexico.

\section{Codex Nuttall}

1992 éd. Ferdinand Anders, Maarten Jansen et Gabina Aurora Pérez Jíménez, ADV/FCE, Graz/Mexico. 
Codex Porfirio Díaz

1892 in Antigüedades Mexicanas, vol. I, Ignacio Chavero, Mexico.

Codex Telleriano-Remensis

1964 in Antigüedades de México, I, 152-336, commentaire de José Corona Nuñez, Secretaría de Hacienda y Crédito Público, Mexico.

Codex Vaticano-Ríos (3738)

1979 Akademische Druck- u. Verlagsanstalt, Graz.

\section{Códices cuicatecos}

2001 éd. Sebastian van Doesburg, Gobierno Constitucional del Estado de Oaxaca, Porrúa, Mexico.

DAvis Irvine

1979 " The Kiowa-Tanoan, Keresan and Zuni Languages », in Lyle Campbell et Marianne Mithun (éds), The Languages of Native America : historical and comparative assessment, University of Texas Press, Austin, pp. 390-443.

Díaz del Castillo Bernal

1977 Historia verdadera de la Conquista de la Nueva España, 2 vols, Porrúa, Mexico.

Dorsey George A.

1904 Traditions of the Skidi Pawnee, Memoirs of the American Folklore Society, Houghton, Mifflin and Company, Boston.

DURÁn fr. Diego

1967 Historia de las Indias de Nueva España e islas de tierra firme, Porrúa, 2 vols, Mexico.

DYER Joseph O.

1917 The Lake Charles Atakapas Cannibals, period of 1817-1820, [s. n.], Galveston.

FowLer William R. Jr.

1989 The Cultural evolution of Ancient Nahua civilizations The Pipil-Nicarao of Central America, University of Oklahoma Press, Norman.

\section{GriFFIN-Pierce Trudy}

1995 The Encyclopedia of Native America, Rushfield Books, Knoxfield.

GuERNSEY Samuel James

1931 " Explorations in Northern Arizona report on the archaeological fieldwork of 1920-1923 ", in Papers of the Peabody Museum of American Archaeology and Ethnology, XII (1), Harvard University, Cambridge.

Historia de los mexicanos por sus pinturas

1988 traduction et étude de Paule Obadia-Baudesson, OxomocoCipactonal, Paris. 


\section{Historia tolteca-chichimeca}

1976 éd. et trad. Paul Kirchhoff, Lina Odena Guëmes et Luis Reyes García, Instituto Nacional de Antropología e Historia/Centro de Investigaciones Superiores del INAH, Secretaría de Educación Pública, Mexico.

Huizinga Johan

1988 Homo ludens. Essai sur la fonction sociale du jeu, Gallimard, Paris [1951].

Jenkins John Holland (éd.)

1958 Recollections of early Texas, University of Texas Press, Austin.

KANTNER John

1999 «Survival cannibalism or sociopolitical intimidation? Explaining perimortem mutilation in the American Southwest ", Human Nature, 10, pp. 1-50.

2004 Ancient Puebloan Southwest, Cambridge University Press, Cambridge.

KIDDER Alfred Vincent

1963 An introduction to the study of Southwestern archaeology, Yale Paperbound, Yale [1924].

KIDDER Alfred Vincent et Samuel J. GuernSEY

1919 Archaeological explorations in Northeastern Arizona, Smithsonian Institution Bureau of American Ethnology, Bulletin 65, Washington.

KNOWLes Nathaniel

1985 " The torture of captives by the Indians of Eastern North America », in Scalping and torture warfares practices among North American Indians, Indian Reprints Iroqrafts, Ohsweken, pp. 195-225 [1940].

Lastra de SuÁrez Yolanda et Fernando Horcasitas

1983 «La lengua náhuatl de México », in América Latina en sus lenguas indigenas, UNESCO/Monte Ávila Editores, Caracas.

León Portilla Miguel

1972 Religión de los Nicaraos. Análisis y comparación de tradiciones culturales nahuas, Universidad Nacional Autónoma de México, Instituto de Investigaciones Históricas, Mexico.

LiNTON Ralph

1926 "The origin of the Skidi Pawnee sacrifice to the Morning Star », American Anthropologist, n. s., 28 (3), pp. 457-466.

LowIE Robert

1982 Indians of the Plains, University of Nebraska Press, Lincoln.

MONTGOMERY H.

1894 "Prehistoric man in Utah », The Archaeologist, 2 (8, 10, 11), pp. 225-234, 298-306, 335-342, Waterloo, Ind.

Motolinía fr. Toribio de

1996 Memoriales, édition critique de Nancy Joe Dyer, El Colegio de México, Mexico. 
Newcomb William W. Jr

1984 The Indians of Texas, University of Texas Press, Austin.

Primeros Memoriales

1905 Estampa I, Paso y Troncoso, vol. VI, Hauser y Menet, Madrid.

ROBERTS Frank

1993 "Cannibalism », American Journal of Physical Anthropology, 91 (4), pp. 421-439.

Rowley MOORe Guy

1939 «Pawnee Traditions and Customs», Chronicles of Oklahoma, 17 (2), pp. 151-169, Oklahoma City.

Seler Eduard

1960 Gesammelte Abhandlungen zur Amerikanischen Sprach- und Altertumskunde, Akademische Druck- u. Verlagsanstalt, vol. II, 402, Graz.

SoLIs fr. Gaspar José de

1931 " Diary of a visit of inspection of the Texas Missions made by fray Gaspar José de Solis in the year 1767-1768 ", translated by Margaret K. Kress, Southwestern Historical Quarterly, 35, pp. 28-76, Austin.

TORQuemada Fr. Juan de

1969 Monarquía Indiana, 3 vols, Porrúa, México.

TURner Christy

1993 «Cannibalism in Chaco Canyon : the charnel pit excavated in 1926 at Small House ruin by Frank H. H. Roberts, Jr. ", American Journal of Physical Anthropology, 91 (4), pp. 421-439.

TURner Christy et Jacqueline TURner

1999 Man corn : cannibalism and violence in the Prehistoric American Southwest, University of Utah Press, Salt Lake City.

VALERI Valerio

1994 "Wild victims : hunting as sacrifice and sacrifice as hunting in Huaulu », History of Religions, 34 (2), pp. 101-131, The University of Chicago Press, Chicago.

Vié-WoHrER Anne-Marie

1999 Xipe Totec, Notre Seigneur l'Écorché. Étude glyphique d'un dieu aztèque, 2 vols, CEMCA, Mexico.

WeDEL Waldo R.

1977 " Native Astronomy and the Plains Caddoans », in Anthony F. Aveni (éd.), Native American astronomy, University of Texas Press, Austin, pp. 131-145.

WHITE Tim D.

1992 Prehistoric cannibalism at Mancos, Princeton University Press, Princeton.

WILLEY Gordon R.

1966 An introduction to american archaeology. 1. North and Middle America, Prentice Hall, Englewood Cliffs. 